\title{
The mean value of the product of class numbers of paired quadratic fields, II
}

\author{
By Anthony C. Kable and Akihiko YukIE
}

(Received Sept. 13, 2000)

(Revised Apr. 4, 2002)

\begin{abstract}
Let $k$ be a number field and $\tilde{k}$ a fixed quadratic extension of $k$. In this paper and its companions, we find the mean value of the product of class numbers and regulators of two quadratic extensions $F, F^{*} \neq \tilde{k}$ contained in the biquadratic extensions of $k$ containing $\tilde{k}$.
\end{abstract}

\section{Introduction.}

This is part II of a series of three papers. We first recall the main result of this series of papers. If $k$ is a number field, let $\Delta_{k}, h_{k}$, and $R_{k}$ be the absolute discriminant (which is an integer), the class number, and the regulator, respectively. We fix a number field $k$ and a quadratic extension $\tilde{k}$ of $k$. If $F \neq \tilde{k}$ is another quadratic extension of $k$, let $\tilde{F}$ be the compositum of $F$ and $\tilde{k}$. Then $\tilde{F}$ is a biquadratic extension of $k$ and so contains precisely three quadratic extensions, $\tilde{k}, F$ and, say, $F^{*}$ of $k$. We say that $F$ and $F^{*}$ are paired.

For simplicity we specialize to the case $k=\boldsymbol{Q}$. Let $\tilde{k}=\boldsymbol{Q}\left(\sqrt{d_{0}}\right)$ where $d_{0} \neq 1$ is a square free integer. Suppose $\left|\Delta_{\boldsymbol{Q}\left(\sqrt{d_{0}}\right)}\right|=\prod_{p} p^{\tilde{\delta}_{p}\left(d_{0}\right)}$ is the prime decomposition. For any prime number $p$, we put

$$
E_{p}^{\prime}\left(d_{0}\right)= \begin{cases}1-3 p^{-3}+2 p^{-4}+p^{-5}-p^{-6} & \text { if } p \text { is split in } \tilde{k}, \\ \left(1+p^{-2}\right)\left(1-p^{-2}-p^{-3}+p^{-4}\right) & \text { if } p \text { is inert in } \tilde{k}, \\ \left(1-p^{-1}\right)\left(1+p^{-2}-p^{-3}+p^{\left.-2 \tilde{\delta}_{p}\left(d_{0}\right)-2\left\lfloor\tilde{\delta}_{p}\left(d_{0}\right) / 2\right\rfloor-1\right)}\right) & \text { if } p \text { is ramified in } \tilde{k},\end{cases}
$$

where $\left\lfloor\tilde{\delta}_{p}\left(d_{0}\right) / 2\right\rfloor$ is the largest integer less than or equal to $\tilde{\delta}_{p}\left(d_{0}\right) / 2$. We define

$$
\begin{aligned}
& c_{+}\left(d_{0}\right)=\left\{\begin{array}{ll}
16 & d_{0}>0, \\
8 \pi & d_{0}<0,
\end{array} \quad c_{-}\left(d_{0}\right)= \begin{cases}4 \pi^{2} & d_{0}>0, \\
8 \pi & d_{0}<0,\end{cases} \right. \\
& M\left(d_{0}\right)=\left|\Delta_{\boldsymbol{Q}\left(\sqrt{d_{0}}\right)}\right|^{1 / 2} \zeta_{\boldsymbol{Q}\left(\sqrt{d_{0}}\right)}(2) \prod_{p} E_{p}^{\prime}\left(d_{0}\right) .
\end{aligned}
$$

The following two theorems are the main results of this series of papers.

2000 Mathematics Subject Classification. Primary 11S90; Secondary 11R45.

Key Words and Phrases. density theorem, prehomogeneous vector spaces, binary Hermitian forms, local zeta functions.

The first author was partially supported by a Dean's incentive Grant, College of Arts and Sciences, Oklahoma State University.

The second author was partially supported by Grant-in-Aid for Scientific Research (No. 1244002), the Ministry of Education, Culture, Sports, Science and Technology, Japan. 
THEOREM 1.1. With either choice of sign we have

$$
\lim _{X \rightarrow \infty} X^{-2} \sum_{\substack{[F: Q]=2, 0< \pm \Delta_{F}<X}} h_{F} R_{F} h_{F^{*}} R_{F^{*}}=c_{ \pm}\left(d_{0}\right)^{-1} M\left(d_{0}\right) .
$$

THEOREM 1.2. With either choice of sign we have

$$
\lim _{X \rightarrow \infty} X^{-2} \sum_{\substack{[F: \boldsymbol{Q}]=2, 0< \pm A_{F}<X}} h_{F\left(\sqrt{d_{0}}\right)} R_{F\left(\sqrt{d_{0}}\right)}=c_{ \pm}\left(d_{0}\right)^{-1} h_{\boldsymbol{Q}\left(\sqrt{d_{0}}\right)} R_{\boldsymbol{Q}\left(\sqrt{d_{0}}\right)} M\left(d_{0}\right) .
$$

For a general introduction to this problem, the reader should see the introduction to Part I. Throughout this part, $k$ is a number field and $\tilde{k}$ is a fixed quadratic extension of $k$ unless otherwise stated. Let $W$ be the space of binary Hermitian forms. Our approach to the above theorems is based on a consideration of the zeta function for the following prehomogeneous vector space:

(1) $G=G L(2) \times G L(2) \times G L(2), V=\mathrm{M}(2,2) \otimes \mathrm{Aff}^{2}$,

(2) $G=G L(2)_{\tilde{k}} \times G L(2), V=W \otimes \mathrm{Aff}^{2}$

where $G L(2)_{\tilde{k}}$ is regarded as a group over $k$. In both cases there exists a relative invariant polynomial $P(x)$ of degree four and we put $V^{\text {ss }}=\{x \in V \mid P(x) \neq 0\}$.

Let $v$ be a place of $k$, and $k_{v}$ be the completion of $k$ at this place. We denote the integer ring of $k_{v}$ by $\mathcal{O}_{v}$. Let $K_{v}$ be the standard maximal compact subgroup of $G_{k_{v}}$. In this part, we only consider $\left(G_{k_{v}}, V_{k_{v}}\right)$ for the above prehomogeneous vector spaces and so the consideration in this part is of a purely local nature.

In Part I, we formulated the filtering process required to prove Theorems 1.1 and 1.2. The only remaining task is to compute the local densities. In this part we compute the local densities for all non-dyadic cases and for those dyadic cases that can be dealt with in a similar manner. However, the computations of the local densities that involve wild ramification at dyadic places are significantly more elaborate and so we carry out these computations separately in Part III, on account of their length. We shall point out which cases are postponed to Part III later in this introduction.

In Part I we selected standard representatives for the orbits in $G_{k_{v}} \backslash V_{k_{v}}^{\text {ss }}$ and introduced an equivalence relation $\asymp$ on $V_{k_{v}}^{\text {ss }}$ whose equivalence classes are unions of $G_{k_{v}}$ orbits. These definitions will be reviewed in Section 2. If $v$ is a finite place, then we use the measure on $V_{k_{v}}$ such that $\operatorname{vol}\left(V_{\mathcal{O}_{v}}\right)=1$ and the measure on $G_{y k_{v}}^{\circ}$ defined in Definition 5.13 of Part I. The measure on $G_{y k_{v}}^{\circ}$ will be reviewed in Section 2.

If $v$ is a finite place, we define

$$
\varepsilon_{v}(x)=\operatorname{vol}\left(K_{v} \cap G_{x k_{v}}^{\circ}\right) \operatorname{vol}\left(K_{v} x\right) .
$$

We put

$$
\bar{\varepsilon}_{v}(x)=\sum_{y \asymp x} \operatorname{vol}\left(K_{v} \cap G_{y k_{v}}^{\circ}\right) \operatorname{vol}\left(K_{v} y\right),
$$

where the sum is over standard representatives for orbits in the equivalence class of $x$. The local density at $v$ is then 


$$
E_{v}=\sum_{x} \varepsilon_{v}(x)=\sum_{x} \bar{\varepsilon}_{v}(x),
$$

where the first sum is over all standard representatives for orbits in $G_{k_{v}} \backslash V_{k_{v}}^{\text {ss }}$ and the second over a set containing one standard representative for an orbit in each class in $G_{k_{v}} \backslash V_{k_{v}}^{\text {ss }} / \asymp$. We have established in Theorem 6.22 and Lemma 7.3 in Part I that $E_{v}$ is the Euler factor at the place $v$ in the Euler product on the right hand side of the equations in Theorems 1.1 and 1.2.

The values of $\bar{\varepsilon}_{v}(x)$ calculated in this part are summarized in Tables 1, 2 and 3. The notation used in the tables will be explained in Section 2. This leaves us the cases $(\mathrm{rm} \mathrm{rm})^{*},(\mathrm{rm} \mathrm{rm} \mathrm{ur})$ and (rm rm rm) (these notation will also be explained in Section 2) for dyadic places, which are dealt with in Part III.

The values of $\operatorname{vol}\left(K_{v} \cap G_{x k_{v}}^{\circ}\right)$ used to determine the entries in the three tables are determined in Propositions 3.2, 3.3, 3.5, 3.6, and the values of $\operatorname{vol}\left(K_{v} x\right)$ in Propositions 4.14, 4.15, 4.25 and 4.26.

Table 1. $\varepsilon_{v}(x)$ for $v$ finite and non-dyadic.

\begin{tabular}{|c|c|}
\hline Index & $\varepsilon_{v}(x)$ \\
\hline \hline$(\mathrm{sp})$ & $\left(1+q_{v}^{-1}\right)\left(1-q_{v}^{-2}\right)^{2} / 2$ \\
\hline$(\mathrm{in})$ & $\left(1-q_{v}^{-1}\right)\left(1-q_{v}^{-4}\right) / 2$ \\
\hline$(\mathrm{rm})$ & $\left(1-q_{v}^{-2}\right)^{2} / 2$ \\
\hline$(\mathrm{sp} \mathrm{ur})$ & $\left(1-q_{v}^{-1}\right)^{3}\left(1-q_{v}^{-2}\right) / 2$ \\
\hline$(\mathrm{sp} \mathrm{rm})$ & $q_{v}^{-1}\left(1-q_{v}^{-1}\right)\left(1-q_{v}^{-2}\right)^{3} / 2$ \\
\hline$($ in ur $)$ & $\left(1-q_{v}^{-1}\right)\left(1-q_{v}^{-4}\right) / 2$ \\
\hline$($ in rm $)$ & $q_{v}^{-1}\left(1-q_{v}^{-1}\right)\left(1-q_{v}^{-2}\right)\left(1-q_{v}^{-4}\right) / 2$ \\
\hline$(\mathrm{rm} \mathrm{ur})$ & $\left(1-q_{v}^{-1}\right)^{2}\left(1-q_{v}^{-2}\right) / 2$ \\
\hline$(\mathrm{rm} \mathrm{rm}) *$ & $q_{v}^{-2}\left(1-q_{v}^{-2}\right)^{2} / 2$ \\
\hline$(\mathrm{rm} \mathrm{rm} \mathrm{ur})$ & $q_{v}^{-2}\left(1-q_{v}^{-1}\right)^{2}\left(1-q_{v}^{-2}\right) / 2$ \\
\hline
\end{tabular}

Table 2. $\varepsilon_{v}(x)$ for ungrouped dyadic orbits.

\begin{tabular}{|c|c|}
\hline Index & $\varepsilon_{v}(x)$ \\
\hline \hline$(\mathrm{sp})$ & $\left(1+q_{v}^{-1}\right)\left(1-q_{v}^{-2}\right)^{2} / 2$ \\
\hline (in) & $\left(1-q_{v}^{-1}\right)\left(1-q_{v}^{-4}\right) / 2$ \\
\hline (rm) & $\left(1-q_{v}^{-2}\right)^{2} / 2$ \\
\hline (sp ur) & $\left(1-q_{v}^{-1}\right)^{3}\left(1-q_{v}^{-2}\right) / 2$ \\
\hline (in ur) & $\left(1-q_{v}^{-1}\right)\left(1-q_{v}^{-4}\right) / 2$ \\
\hline (rm ur) & $\left(1-q_{v}^{-1}\right)^{2}\left(1-q_{v}^{-2}\right) / 2$ \\
\hline
\end{tabular}


Table 3. $\bar{\varepsilon}_{v}(x)$ for grouped dyadic orbits.

\begin{tabular}{|c|c|c|}
\hline Index & Conditions & $\bar{\varepsilon}_{v}(x)$ \\
\hline \hline (sp rm) & $\delta_{x, v} \leq 2 m_{v}$ & $q_{v}^{-\delta_{x, v} / 2}\left(1-q_{v}^{-1}\right)^{2}\left(1-q_{v}^{-2}\right)^{3}$ \\
\hline (sp rm) & $\delta_{x, v}=2 m_{v}+1$ & $q_{v}^{-\left(m_{v}+1\right)}\left(1-q_{v}^{-1}\right)\left(1-q_{v}^{-2}\right)^{3}$ \\
\hline (in rm) & $\delta_{x, v} \leq 2 m_{v}$ & $q_{v}^{-\delta_{x, v} / 2}\left(1-q_{v}^{-1}\right)^{2}\left(1-q_{v}^{-2}\right)\left(1-q_{v}^{-4}\right)$ \\
\hline (in rm) & $\delta_{x, v}=2 m_{v}+1$ & $q_{v}^{-\left(m_{v}+1\right)}\left(1-q_{v}^{-1}\right)\left(1-q_{v}^{-2}\right)\left(1-q_{v}^{-4}\right)$ \\
\hline
\end{tabular}

We shall review and compute the local densities at the infinite places in Section 5. The method we use is to reduce the problem to the computation of the Jacobian determinants of certain maps. The contributions from the various orbits are given in Propositions 5.2, 5.4, 5.6 and 5.7.

Even though we follow the notation and definitions in Part I, a minimal review of the basic concepts and definitions should help the reader and we shall provide this in Section 2. In Sections 3 and 4 we compute the values of $\operatorname{vol}\left(K_{v} \cap G_{x k_{v}}^{\circ}\right)$ and $\operatorname{vol}\left(K_{v} x\right)$ for orbits listed in Tables 1, 2 and 3. In Section 5, we find the local densities at the infinite places.

\section{Review of facts from Part I.}

In this section we give a minimal review of basic notation and definitions from Part I that are needed in this part.

If $X$ is a finite set then $\# X$ will denote its cardinality. The standard symbols $\boldsymbol{Q}, \boldsymbol{R}$, $C$ and $Z$ will denote respectively the rational, real and complex numbers and the rational integers. If $R$ is any ring then $R^{\times}$is the set of invertible elements of $R$ and if $V$ is a variety defined over $R$ then $V_{R}$ denotes its $R$-points. If $G$ is an algebraic group then $G^{\circ}$ denotes its identity component.

Throughout this paper, $k$ is a fixed number field and $\tilde{k}$ is a fixed quadratic extension of $k$, unless otherwise stated.

Let $\mathfrak{M}, \mathfrak{M}_{\infty}, \mathfrak{M}_{\mathrm{f}}, \mathfrak{M}_{\mathrm{dy}}, \mathfrak{M}_{\boldsymbol{R}}$ and $\mathfrak{M}_{\boldsymbol{C}}$ denote respectively the set of all places of $k$, all infinite places, all finite places, all dyadic places (those dividing the place of $\boldsymbol{Q}$ at 2), all real places and all complex places. (Correspondingly we have $\tilde{\mathfrak{M}}$ and so on.) Let $\mathfrak{M}_{\text {rm }}, \mathfrak{M}_{\text {in }}$ and $\mathfrak{M}_{\text {sp }}$ be the sets of places of $k$ which are respectively ramified, inert and split on extension to $\tilde{k}$. Recall that a real place of $k$ which lies under a complex place of $\tilde{k}$ is regarded as ramified.

We denote the absolute value in $k_{v}$ by ||$_{v}$. If $v \in \mathfrak{M}_{\mathrm{f}}$, let $\mathcal{O}_{v}$ be the integer ring of $k_{v}$ and $\mathfrak{p}_{v}=\left(\pi_{v}\right)$ be its prime ideal. Let $q_{v}=\# \mathcal{O}_{v} / \mathfrak{p}_{v}$.

As far as notation pertaining to number fields and local fields, we use the same conventions as in Part I: the notation for the $\tilde{k}$ object will be derived from that of the $k$ object by adding a tilde and, for other fields, by writing the field in question as the subscript. For example, $\mathcal{O}_{F}$ for the ring of integers of the field $F$. If $a \in k_{v}$ and $(a)=\mathfrak{p}_{v}^{i}$ then we write $\operatorname{ord}_{k_{v}}(a)=i$. If $\mathfrak{i}$ is a fractional ideal in $k_{v}$ and $a-b \in \mathfrak{i}$ then we write $a \equiv b(\mathfrak{i})$ or $a \equiv b(c)$ if $c$ generates $\mathfrak{i}$. 
If $k_{1} / k_{2}$ is a finite extension either of local fields or of number fields then we shall write $\Delta_{k_{1} / k_{2}}$ for the relative discriminant of the extension; it is an ideal in the ring of integers of $k_{2}$. We put $\Delta_{\tilde{k}_{v} / k_{v}}=\mathfrak{p}_{v}^{\tilde{\delta}_{v}}$ if $v \in \mathfrak{M}_{\mathrm{f}}$ and $\tilde{k}_{v}=\tilde{k} \otimes k_{v}$ is a field. We shall use the notation $\operatorname{Tr}_{k_{1} / k_{2}}$ and $\mathrm{N}_{k_{1} / k_{2}}$ for the trace and the norm in the extension $k_{1} / k_{2}$.

We assume that the reader is familiar with the basic definitions and facts concerning local fields. These may be found in [5]. We choose Haar measures $d x_{v}$ on $k_{v}$ and $d^{\times} t_{v} k_{v}^{\times}$so that $\int_{\mathcal{O}_{v}} d x_{v}=1$ and $\int_{\mathcal{O}_{v}^{\times}} d^{\times} t_{v}=1$.

Let $k$ be an arbitrary field of characteristic zero, and $\tilde{k}$ be a fixed quadratic extension of $k$. We denote the non-identity element of $\operatorname{Gal}(\tilde{k} / k)$ by $\sigma$. Let $(G, V)$ be either the prehomogeneous vector space (1) or (2) in the introduction.

If $g \in G$ then we shall write $g=\left(g_{1}, g_{2}, g_{3}\right)$ in case (1) and $g=\left(g_{1}, g_{2}\right)$ in case (2). It will be convenient to identify $x=\left(x_{1}, x_{2}\right) \in V$ with the $2 \times 2$-matrix $M_{x}(v)=$ $v_{1} x_{1}+v_{2} x_{2}$ of linear forms in the variables $v_{1}$ and $v_{2}$, which we collect into the row vector $v=\left(v_{1}, v_{2}\right)$. With this identification, we define a rational action of $G$ on $V$ via

$$
M_{g x}(v)= \begin{cases}g_{1} M_{x}\left(v g_{3}\right)^{t} g_{2} & \text { in case }(1), \\ g_{1} M_{x}\left(v g_{2}\right)^{t} g_{1}^{\sigma} & \text { in case }(2) .\end{cases}
$$

In both cases we define $F_{x}(v)=-\operatorname{det} M_{x}(v)$. Then

$$
F_{g x}(v)= \begin{cases}\operatorname{det} g_{1} \operatorname{det} g_{2} F_{x}\left(v g_{3}\right) & \text { in case }(1), \\ \mathrm{N}_{\tilde{k} / k}\left(\operatorname{det} g_{1}\right) F_{x}\left(v g_{2}\right) & \text { in case }(2)\end{cases}
$$

We let $P(x)$ be the discriminant of the binary quadratic form $F_{x}(v)$. Then $P(x) \in k[V]$ and $P(g x)=\chi(g) P(x)$ where

$$
\chi(g)= \begin{cases}\left(\operatorname{det} g_{1} \operatorname{det} g_{2} \operatorname{det} g_{3}\right)^{2} & \text { in case }(1), \\ \left(\mathbf{N}_{\tilde{k} / k}\left(\operatorname{det} g_{1}\right) \operatorname{det} g_{3}\right)^{2} & \text { in case }(2) .\end{cases}
$$

The polynomial $P(x)$ is not identically zero and we write $V^{\text {ss }}=\{x \in V \mid P(x) \neq 0\}$. As $\tilde{k}$-varieties,

$$
G \times \tilde{k} \cong G L(2) \times G L(2) \times G L(2)
$$

and

$$
W \times \tilde{k} \cong \mathrm{M}(2,2)
$$

so that

$$
V \times \tilde{k} \cong \mathrm{M}(2,2) \otimes \mathrm{Aff}^{2},
$$

and a calculation shows that the induced action of $G \times \tilde{k}$ on $V \times \tilde{k}$ is that of case (1). The Galois automorphism $\sigma$ induces a $k$-automorphism of the $k$-varieties $G$ and $V$ which we denote by $i(\sigma)$. If $\left(g_{1}, g_{2}, g_{3}\right) \in G_{\tilde{k}}$ then $i(\sigma)\left(g_{1}, g_{2}, g_{3}\right)=\left(g_{2}^{\sigma}, g_{1}^{\sigma}, g_{3}^{\sigma}\right)$ and if $x \in W_{\tilde{k}}$ then $i(\sigma) x={ }^{t} x^{\sigma}$, where $\sigma$ as a superscript denotes the entry-by-entry action of $\sigma$. In particular, $G_{k}$ is embedded in $G_{\tilde{k}} \cong(G \times \tilde{k})_{\tilde{k}}$ via the map $\left(g_{1}, g_{2}\right) \mapsto\left(g_{1}, g_{1}^{\sigma}, g_{2}\right)$.

It is proved in [6], pp. 305-310 and [1], p. 324 that $G_{k} \backslash V_{k}^{\text {ss }}$ corresponds bijectively with isomorphism classes of field extensions of $k$ of degree one or two. Moreover if $x \in V$ then the corresponding field is generated by the roots of $F_{x}(v)=0$. We denote this field by $k(x)$. 
Suppose that $p(z)=z^{2}+a_{1} z+a_{2} \in k[z]$ has distinct roots $\alpha_{1}$ and $\alpha_{2}$. We collect these into a set $\alpha=\left\{\alpha_{1}, \alpha_{2}\right\}$ since the numbering is arbitrary. Define $w_{p} \in V_{k}$ by

$$
w_{p}=\left(\left(\begin{array}{cc}
0 & 1 \\
1 & a_{1}
\end{array}\right),\left(\begin{array}{cc}
1 & a_{1} \\
a_{1} & a_{1}^{2}-a_{2}
\end{array}\right)\right)
$$

a computation shows that $F_{w_{p}}(z, 1)=p(z)$ and so $w_{p} \in V_{k}^{\text {ss }}$ and $k\left(w_{p}\right)=k(\alpha)$ is the splitting field of $p$. We can choose a representative of the form $w_{p}$ for each orbit in the orbit space $G_{k_{v}} \backslash V_{k_{v}}^{\text {ss }}$. These are the standard representatives.

Let

$$
\begin{aligned}
w & =\left(\left(\begin{array}{ll}
1 & 0 \\
0 & 0
\end{array}\right),\left(\begin{array}{ll}
0 & 0 \\
0 & 1
\end{array}\right)\right), \\
h_{\alpha} & =\left(\begin{array}{cc}
1 & -1 \\
-\alpha_{1} & \alpha_{2}
\end{array}\right)
\end{aligned}
$$

and then define $g_{p} \in G_{k\left(w_{p}\right)}$ by

$$
g_{p}= \begin{cases}\left(h_{\alpha}, h_{\alpha},\left(\alpha_{2}-\alpha_{1}\right)^{-1} h_{\alpha}\right) & \text { in case }(1) \text { or when } k\left(w_{p}\right)=\tilde{k}, \\ \left(h_{\alpha},\left(\alpha_{2}-\alpha_{1}\right)^{-1} h_{\alpha}\right) & \text { otherwise. }\end{cases}
$$

With these definitions it is easy to check that $w_{p}=g_{p} w$.

We recall two propositions regarding the explicit description of how $G_{w_{p} k}$ is embedded in $G_{k}$ in each case. Let

$$
A_{p}(c, d)=\left(\begin{array}{cc}
c & -d \\
a_{2} d & c-a_{1} d
\end{array}\right) \text { and } \tau_{p}=\left(\begin{array}{cc}
-1 & 0 \\
-a_{1} & 1
\end{array}\right) .
$$

Then the following are Lemmas 3.27, 3.30 of Part I.

LEMMA 2.12. In case (1), $G_{w_{p} k}^{\circ}$ consists of elements of $G_{k}$ of the form

$$
\left(A_{p}\left(c_{1}, d_{1}\right), A_{p}\left(c_{2}, d_{2}\right), A_{p}\left(c_{3}, d_{3}\right)\right)
$$

where $c_{i}, d_{i} \in k, \operatorname{det}\left(A_{p}\left(c_{i}, d_{i}\right)\right) \neq 0$ for $i=1,2$ and $\left(c_{3}, d_{3}\right)$ is related to $\left(c_{1}, d_{1}, c_{2}, d_{2}\right)$ by the equation

$$
A_{p}\left(c_{3}, d_{3}\right)=A_{p}\left(c_{1}, d_{1}\right)^{-1} A_{p}\left(c_{2}, d_{2}\right)^{-1} \text {. }
$$

Moreover $\left[G_{w_{p} k}: G_{w_{p} k}^{\circ}\right]=2$ and $G_{w_{p} k} / G_{w_{p} k}^{\circ}$ is generated by the class of $\left(\tau_{p}, \tau_{p}, \tau_{p}\right)$.

LEMMA 2.15. In case (2), $G_{w_{p} k}^{\circ}$ consists of elements of $G_{k}$ of the form

$$
\left(A_{p}\left(c_{1}, d_{1}\right), A_{p}\left(c_{2}, d_{2}\right)\right)
$$

where $c_{1}, d_{1} \in \tilde{k}, c_{2}, d_{2} \in k, \operatorname{det}\left(A_{p}\left(c_{1}, d_{1}\right)\right) \neq 0$ and $\left(c_{2}, d_{2}\right)$ is related to $\left(c_{1}, d_{1}\right)$ by the equation

$$
A_{p}\left(c_{2}, d_{2}\right)=A_{p}\left(c_{1}, d_{1}\right)^{-1} A_{p}\left(c_{1}^{\sigma}, d_{1}^{\sigma}\right)^{-1} \text {. }
$$

Moreover, $\left[G_{w_{p} k}: G_{w_{p} k}^{\circ}\right]=2$ and $G_{w_{p} k} / G_{w_{p} k}^{\circ}$ is generated by the class of $\left(\tau_{p}, \tau_{p}\right)$.

We now assume that $k$ is a number field again. Let $v \in \mathfrak{M}_{\mathrm{f}}$. Recall that $p(z) \in$ $k_{v}[z]$ is called an Eisenstein polynomial if $a_{1} \in \mathfrak{p}_{v}$ and $a_{2} \in \mathfrak{p}_{v} \backslash \mathfrak{p}_{v}^{2}$. If $F / k_{v}$ is a ramified 
extension then there is always an Eisenstein polynomial whose roots generate $F$ over $k_{v}$ and any such polynomial will satisfy a condition that either of its roots generates the integer ring of a ramified quadratic extension of $k_{v}$. For each $v \in \mathfrak{M}_{\mathrm{f}}, k_{v}$ has a unique unramified quadratic extension. If $F$ is this extension and $v \notin \mathfrak{M}_{\mathrm{dy}}$ then the integer ring of $F$ is generated by a root of $p(z)$ with $a_{1}=0$ and $-a_{2}$ any non-square unit in $k_{v}$. If $v \in \mathfrak{M}_{\mathrm{dy}}$ then we must instead take $p(z)$ to be an Artin-Schreier polynomial, which means, by definition, that $p(z)$ is irreducible in $k_{v}[z], a_{1}=-1$ and $a_{2}$ is a unit. Note that $p$ stays irreducible modulo $\mathfrak{p}_{v}$ in this case by Hensel's lemma.

For each $v \in \mathfrak{M}_{\mathrm{f}}$ we choose a list of representatives $w_{v, 1}, \ldots, w_{v, N_{v}}$, one for each of the $G_{k_{v}}$-orbits in $V_{k_{v}}^{\text {ss }}$, in such a way that $P\left(w_{v, i}\right)$ generates the ideal $\Delta_{k\left(w_{v, i}\right) / k_{v}}$ for $i=1, \ldots, N_{v}$. This is possible, in light of the previous paragraph, if we take each $w_{v, i}$ to equal $w_{p}$ for a suitable $p(z) \in k_{v}[z]$. In the special case where $k\left(w_{v}, i\right)=k_{v}$ we take $w_{v, i}=w$ or $w_{p}$ for $p(z)=z^{2}-z$. For $v \in \mathfrak{M}_{\infty}$ we require instead that $\left|P\left(w_{v, i}\right)\right|_{v}=1$ for $i=1, \ldots, N_{v}$, which is clearly possible. In both cases we assume for convenience that $w_{v, 1}$ represents the orbit corresponding to $k_{v}$ itself. If $x$ is a standard representative and $k_{v}(x) \neq k_{v}$ then let $\Delta_{k_{v}(x) / k_{v}}=\mathfrak{p}_{v}^{\delta_{x, v}}$.

Let $v \in \mathfrak{M}$ and $x \in V_{k_{v}}^{\mathrm{ss}}$. If $v \notin \mathfrak{M}_{\mathrm{sp}}$ then $v$ extends uniquely to a place of $\tilde{k}$ which we also denote by $v$. In this case $\tilde{k}_{v} \cong k_{v} \otimes_{k} \tilde{k}$. We denote by $\tilde{k}_{v}(x)$ the compositum of $\tilde{k}_{v}$ and $k_{v}(x)$.

Let $v \in \mathfrak{M}$. We now review the index attached to each orbit in $V_{k_{v}}^{\text {ss }}$, which was introduced in Part I as a bookkeeping device. The orbit corresponding to $k_{v}$ itself will have index (sp), (in), or (rm) according as $v$ is in $\mathfrak{M}_{\mathrm{sp}}, \mathfrak{M}_{\text {in }}$, or $\mathfrak{M}_{\mathrm{rm}}$. The orbit corresponding to the unique unramified quadratic extension of $k_{v}$ will have index (sp ur), (in ur) and (rm ur) for $v \in \mathfrak{M}_{\mathrm{sp}}, v \in \mathfrak{M}_{\text {in }}$ and $v \in \mathfrak{M}_{\mathrm{rm}}$, respectively. An orbit corresponding to a ramified quadratic extension of $k_{v}$ will have index ( $\mathrm{sp} \mathrm{rm}$ ) if $v \in \mathfrak{M}_{\mathrm{sp}}$ and (in $\mathrm{rm}$ ) if $v \in \mathfrak{M}_{\text {in }}$. If $v \in \mathfrak{M}_{\mathrm{rm}}$ then the orbits corresponding to ramified quadratic extensions of $k_{v}$ are subdivided into three types: the one corresponding to $\tilde{k}_{v}$ has index $(\mathrm{rm} \mathrm{rm})^{*}$, those corresponding to quadratic extensions $k_{v}(x) / k_{v}$ such that $k_{v}(x) \neq \tilde{k}_{v}$ and $\tilde{k}_{v}(x) / \tilde{k}_{v}$ is unramified have index ( $\mathrm{rm}$ rm ur) and those corresponding to quadratic extensions $k_{v}(x) / k_{v}$ such that $k_{v}(x) \neq \tilde{k}_{v}$ and $\tilde{k}_{v}(x) / \tilde{k}_{v}$ is ramified have index (rm rm rm). This last index can occur only if $v \in \mathfrak{M}_{\mathrm{dy}}$. The type of $x \in V_{k_{v}}^{\mathrm{ss}}$ will be the index attached to the orbit $G_{k_{v}} x$.

If $v \notin \mathfrak{M}_{\mathrm{dy}}$ or if $v \in \mathfrak{M}_{\mathrm{dy}}$ but $k_{v}(x) / k_{v}$ is unramified (including the case $k_{v}(x)=k_{v}$ ) then we shall write $x \asymp y$ if and only if $y \in G_{k_{v}} x$. Suppose now that $v \in \mathfrak{M}_{\mathrm{dy}}$ and that $k_{v}(x) / k_{v}$ is ramified. If the type of $x$ is (sp $\mathrm{rm}$ ) or (in $\mathrm{rm}$ ) then we shall write $x \asymp y$ if and only if $\Delta_{k_{v}(x) / k_{v}}=\Delta_{k_{v}(y) / k_{v}}$. In this part we do not consider orbits with indices $(\mathrm{rm} \mathrm{rm})^{*},(\mathrm{rm} \mathrm{rm}$ ur) or $(\mathrm{rm} \mathrm{rm} \mathrm{rm})$ for dyadic places and so we shall not recall the definition of $\asymp$ for such orbits. It may be found in Section 7 of Part I and, in a different but equivalent formulation, near the end of Section 3 of Part III.

As in Part I, we use the following notation

$$
a\left(t_{1}, t_{2}\right)=\left(\begin{array}{cc}
t_{1} & 0 \\
0 & t_{2}
\end{array}\right), \quad n(u)=\left(\begin{array}{ll}
1 & 0 \\
u & 1
\end{array}\right) .
$$

We use coordinate systems on $G$ and $V$ similar to those in Part I, as follows. Elements of $G$ have the form $g=\left(g_{1}, g_{2}, g_{3}\right)$ or $g=\left(g_{1}, g_{2}\right)$. In either case we shall 
write

$$
g_{i}=\left(\begin{array}{ll}
g_{i 11} & g_{i 12} \\
g_{i 21} & g_{i 22}
\end{array}\right)
$$

for each $i$. Elements of $V$ are vectors $x=\left(x_{1}, x_{2}\right)$. We shall put

$$
x_{i}=\left(\begin{array}{ll}
x_{i 11} & x_{i 12} \\
x_{i 21} & x_{i 22}
\end{array}\right)
$$

in case (1) and

$$
x_{i}=\left(\begin{array}{cc}
x_{i 0} & x_{i 1} \\
x_{i 1}^{\sigma} & x_{i 2}
\end{array}\right)
$$

in case (2).

Let $v \in \mathfrak{M}_{\mathrm{f}}$. We now review the parametrization and the measure on the stabilizer. Define

$$
H_{x k_{v}}= \begin{cases}\left(k_{v}^{\times}\right)^{4} & (\mathrm{sp}), \\ \left(k_{v}(x)^{\times}\right)^{2} & (\text { sp ur), (sp rm), } \\ \left(\tilde{k}_{v}^{\times}\right)^{2} & (\text { in }),(\mathrm{rm}),(\text { in ur }),(\mathrm{rm} \mathrm{rm})^{*} \\ \tilde{k}_{v}(x)^{\times} & \text {otherwise },\end{cases}
$$

for each of the various indices then $G_{x k_{v}}^{\circ} \cong H_{x k_{v}}$ in all cases. We may regard $H_{x k_{v}}$ as the $k_{v}$-points of an algebraic group $H_{x}$ defined over $\mathcal{O}_{v}$ and we shall do so below.

If $k_{v}(x) / k_{v}$ is quadratic then we shall write $v$ for the generator of $\operatorname{Gal}\left(k_{v}(x) / k_{v}\right)$. If $\tilde{k}_{v}(x) \neq \tilde{k}_{v}$ then $v$ may also be regarded as the generator of $\operatorname{Gal}\left(\tilde{k}_{v}(x) / \tilde{k}_{v}\right)$.

If $x$ is a point of type (sp), we write

$$
s_{x}\left(t_{x}\right)=\left(a\left(t_{11}, t_{12}\right), a\left(t_{21}, t_{22}\right), a\left(\left(t_{11} t_{21}\right)^{-1},\left(t_{12} t_{22}\right)^{-1}\right)\right),
$$

where $t_{x}=\left(t_{11}, \ldots, t_{22}\right) \in\left(k_{v}^{\times}\right)^{4}$. Let $s_{x 1}\left(t_{x}\right), s_{x 2}\left(t_{x}\right), s_{x 3}\left(t_{x}\right)$ be the three components of $s_{x}\left(t_{x}\right)$. If $x$ is a point of type (sp ur) or ( $\mathrm{sp} \mathrm{rm}$ ), we write

$$
s_{x}\left(t_{x}\right)=\left(a\left(t_{11}, t_{11}^{v}\right), a\left(t_{21}, t_{21}^{v}\right), a\left(\left(t_{11} t_{21}\right)^{-1},\left(t_{11}^{v} t_{21}^{v}\right)^{-1}\right)\right),
$$

where $t_{x}=\left(t_{11}, t_{21}\right) \in\left(k(x)_{v}^{\times}\right)^{2}$. We use the notation $s_{x 1}\left(t_{x}\right)$ et cetera for this case also. If $x$ is a point of type (in) or ( $\mathrm{rm})$ then we write

$$
s_{x}\left(t_{x}\right)=\left(a\left(t_{11}, t_{12}\right), a\left(\mathrm{~N}_{\tilde{k}_{v} / k_{v}}\left(t_{11}^{-1}\right), \mathrm{N}_{\tilde{k}_{v} / k_{v}}\left(t_{12}^{-1}\right)\right),\right.
$$

where $t_{x}=\left(t_{11}, t_{12}\right) \in\left(\tilde{k}_{v}^{\times}\right)^{2}$. We use the notation $s_{x 1}\left(t_{x}\right)$ et cetera for this case also. If $x$ is a point of type (in ur) or $(\mathrm{rm} \mathrm{rm})^{*}$ then we write

$$
s_{x}\left(t_{x}\right)=\left(a\left(t_{11}, t_{12}\right), a\left(t_{12}^{\sigma}, t_{11}^{\sigma}\right), a\left(\left(t_{11} t_{12}^{\sigma}\right)^{-1},\left(t_{11}^{\sigma} t_{12}\right)^{-1}\right)\right),
$$

where $t_{x}=\left(t_{11}, t_{12}\right) \in\left(\tilde{k}_{v}^{\times}\right)^{2}$. We use the notation $s_{x 1}\left(t_{x}\right)$ et cetera for this case also. Finally if $x$ is a point of type (in rm), (rm ur), (rm rm ur), or ( $\mathrm{rm} \mathrm{rm} \mathrm{rm}$ ) then we write

$$
s_{x}\left(t_{x}\right)=\left(a\left(t_{11}, t_{11}^{v}\right), a\left(\mathrm{~N}_{\tilde{k}_{v}(x) / k_{v}(x)}\left(t_{11}^{-1}\right), \mathrm{N}_{\tilde{k}_{v}(x) / k_{v}(x)}\left(t_{11}^{-1}\right)^{v}\right)\right),
$$

where $t_{x}=t_{11} \in \tilde{k}_{v}(x)^{\times}$. We use the notation $s_{x 1}\left(t_{x}\right)$ et cetera for this case also. On $H_{x k_{v}}$ we define an invariant measure $d t_{x, v}$ as follows: 


$$
d t_{x, v}= \begin{cases}d^{\times} t_{11 v} d^{\times} t_{12 v} d^{\times} t_{21 v} d^{\times} t_{22 v} & (\mathrm{sp}), \\ d^{\times} t_{11 v} d^{\times} t_{21 v} & \text { (sp ur), (sp rm), } \\ d^{\times} t_{11 v} d^{\times} t_{12 v} & \text { (in), (rm), (in ur), (rm rm)* } \\ d^{\times} t_{11 v} & \text { otherwise. }\end{cases}
$$

We note that if $v \in \mathfrak{M}_{\mathrm{f}}$ then the volume of $H_{x \mathcal{O}_{v}}$ under this measure is 1 in every case.

Let

$$
\tau=\left(\begin{array}{ll}
0 & 1 \\
1 & 0
\end{array}\right)
$$

Suppose that $x \in V_{k_{v}}^{\mathrm{ss}}$ corresponds to a quadratic extension of $k_{v}$. Then it is possible to choose an element $g_{x} \in G_{k(x)}$ such that $x=g_{x} w$ and

$$
g_{x}^{-1} g_{x}^{v}=(-\tau,-\tau, \tau) \text { or }(-\tau, \tau)
$$

If $x=g_{x} w$ with $g_{x} \in G_{k_{v}}$ then we do not impose any condition on $g_{x}$. Then in all cases

$$
G_{x k_{v}}^{\circ}=g_{x}\left\{s_{x}\left(t_{x}\right) \mid t_{x} \in H_{x k_{v}}\right\} g_{x}^{-1} .
$$

Let $\theta_{g_{x}}: G_{x k_{v}}^{\circ} \rightarrow H_{x k_{v}}$ be the isomorphism given by $\theta_{g_{x}}\left(g_{x} s_{x}\left(t_{x}\right) g_{x}^{-1}\right)=t_{x}$. If $g_{x}$ is obvious from the text, we may simply write $\theta$. We have established immediately before Definition 5.13 of Part I that if $x \in G_{k_{v}} w$ or $k_{v}(x) \neq k_{v}$ and $g_{x}$ satisfies (2.30) then the measure $d g_{x, v}^{\prime \prime}=\theta_{g_{x}}^{*}\left(d t_{x, v}\right)$ on $G_{k_{v}(x)}^{\circ}$ does not depend on the choice of $g_{x}$.

\section{The volume of the integral points of the stabilizer.}

Suppose that $v \in \mathfrak{M}_{\mathrm{f}}$ and that $x \in V_{k_{v}}^{\text {ss }}$ is a standard orbital representative. In this section we shall compute the volume of the intersection $K_{v} \cap G_{x k_{v}}^{\circ}$ under the measure $d g_{x, v}^{\prime \prime}$, unless $v \in \mathfrak{M}_{\mathrm{dy}}$ and $x$ has type (rm rm ur) or (rm rm rm). These cases, which involve additional technicalities, will be dealt with in [3].

As in Section 2, we let $\Delta_{\tilde{k}_{v} / k_{v}}=\mathfrak{p}_{v}^{\tilde{\delta}_{v}}$. We shall use the parametrization of the stabilizer introduced in Section 2. Note that since $w, w_{p} \in V_{\mathcal{V}_{v}}$ in all cases, $G_{w}$ and $G_{w_{p}}$ are defined over $\mathcal{O}_{v}$. The canonical measure on $G_{x k_{v}}^{\circ}$ is induced via the map $\theta: G_{x k_{v}}^{\circ} \rightarrow$ $H_{x k_{v}}$ from the measure $d t_{x, v}$ (we are suppressing the element $g_{x}$ since it plays no essential role here). Now $\theta\left(K_{v} \cap G_{x k_{v}}^{\circ}\right) \subseteq H_{x \mathcal{U}_{v}}$ and the measure of $H_{x \mathcal{O}_{v}}$ under $d t_{x, v}$ is 1 . Below we shall consider the subgroup $\theta\left(K_{v} \cap G_{x k_{v}}^{\circ}\right)$ of $H_{x \mathcal{O}_{v}}$ in order to compute $\operatorname{vol}\left(K_{v} \cap G_{x k_{v}}^{\circ}\right)$.

LEMMA 3.1. Let $x=w_{p}$ with $p(z)=z^{2}+a_{1} z+a_{2}$ be a standard orbital representative. If $v \in \mathfrak{M}_{\mathrm{sp}}$ then $g=\left(A_{p}\left(c_{1}, d_{1}\right), A_{p}\left(c_{2}, d_{2}\right), A_{p}\left(c_{3}, d_{3}\right)\right) \in G_{x k_{v}}^{\circ}$ lies in $K_{v}$ if and only if $c_{1}, c_{2}, d_{1}, d_{2} \in \mathcal{O}_{v}$ and $\operatorname{det} A_{p}\left(c_{j}, d_{j}\right) \in \mathcal{O}_{v}^{\times}$for $j=1,2$. If $v \notin \mathfrak{M}_{\mathrm{sp}}$ then $g=\left(A_{p}\left(c_{1}, d_{1}\right)\right.$, $\left.A_{p}\left(c_{2}, d_{2}\right)\right) \in G_{x k_{v}}^{\circ}$ lies in $K_{v}$ if and only if $c_{1}, d_{1} \in \tilde{\mathcal{O}}_{v}$ and $\operatorname{det} A_{p}\left(c_{1}, d_{1}\right) \in \tilde{\mathcal{O}}_{v}^{\times}$.

Proof. The conditions on $c_{j}, d_{j}$ and $\operatorname{det} A_{p}\left(c_{j}, d_{j}\right)$ which are proposed in the statement simply say that $A_{p}\left(c_{j}, d_{j}\right)$ lies in the standard maximal compact subgroup in that factor for $j=1,2$ (respectively $j=1$ ) and so they are certainly necessary. To see that they are also sufficient we must show that they imply that the last entry in $g$ also lies in the relevant maximal compact subgroup. But this follows immediately from Lemmas 2.12 and 2.15. 
In the following, when we consider $w_{p}$ we let $\alpha=\left\{\alpha_{1}, \alpha_{2}\right\}$ be the set of roots of $p(z)$ as usual. Also, when $k_{v}\left(w_{p}\right) / k_{v}$ is quadratic, we denote by $v$ the non-trivial element of $\operatorname{Gal}\left(k_{v}\left(w_{p}\right) / k_{v}\right)$.

Proposition 3.2. Suppose that the index of the orbit containing the standard representative $x$ is on the list (sp), (in), (rm), (sp ur), (sp rm). Then $\operatorname{vol}\left(K_{v} \cap G_{x k_{v}}^{\circ}\right)=1$.

Proof. We have to show that in every case listed above $\theta\left(K_{v} \cap G_{x k_{v}}^{\circ}\right)=H_{x \mathcal{O}_{v}}$. Of the list (sp), (in), (rm) we deal only with the first, since the other two are very similar. For this orbit, $p(z)=z^{2}-z$, we have $H_{x \mathcal{O}_{v}}=\left(\mathcal{O}_{v}^{\times}\right)^{4}$ and the map $\theta$ is $\theta\left(A_{p}\left(c_{1}, d_{1}\right)\right.$, $\left.A_{p}\left(c_{2}, d_{2}\right), A_{p}\left(c_{3}, d_{3}\right)\right)=\left(c_{1}, c_{1}+d_{1}, c_{2}, c_{2}+d_{2}\right)$. Note that in this case we may choose $h_{\alpha}=\left(\begin{array}{cc}1 & -1 \\ 0 & 1\end{array}\right)$ in (2.10) and then computing $g_{p} s_{x}\left(t_{x}\right) g_{p}^{-1}$ in (2.23) explicitly for $t_{x}=$ $\left(c_{1}, c_{1}+d_{1}, c_{2}, c_{2}+d_{2}\right)$ we get the given formula for $\theta$. If $h=\left(t_{1}, t_{2}, t_{3}, t_{4}\right) \in H_{x \mathcal{U}_{v}}$ then $\theta(g)=h$ where $g=\left(A_{p}\left(t_{1}, t_{2}-t_{1}\right), A_{p}\left(t_{3}, t_{4}-t_{3}\right), *\right)$ and, since $\operatorname{det} A_{p}\left(t_{i}, t_{j}-t_{i}\right)=t_{i} t_{j}$, it follows from Lemma 3.1 that $g \in K_{v}$.

This leaves the cases (sp ur) and (sp rm). Recall that $p(z)$ was chosen so that $\mathcal{O}_{k_{v}(x)}=\mathcal{O}_{v}\left[\alpha_{1}\right]$. We have $H_{x \mathcal{O}_{v}}=\left(\mathcal{O}_{k_{v}(x)}^{\times}\right)^{2}$ and the map $\theta$ is

$$
\theta\left(A_{p}\left(c_{1}, d_{1}\right), A_{p}\left(c_{2}, d_{2}\right), *\right)=\left(c_{1}+d_{1} \alpha_{1}, c_{2}+d_{2} \alpha_{1}\right) \text {. }
$$

If $h=\left(t_{1}, t_{2}\right) \in H_{x \mathcal{O}_{v}}$ then $t_{1}, t_{2} \in \mathcal{O}_{k_{v}(x)}^{\times}$and so we may find $c_{1}, d_{1}, c_{2}, d_{2} \in \mathcal{O}_{v}$ so that $t_{1}=c_{1}+d_{1} \alpha_{1}$ and $t_{2}=c_{2}+d_{2} \alpha_{1}$. If we set $g=\left(A_{p}\left(c_{1}, d_{1}\right), A_{p}\left(c_{2}, d_{2}\right), *\right) \in G_{x k_{v}}^{\circ}$ then $\operatorname{det} A_{p}\left(c_{j}, d_{j}\right)=\left(c_{j}+d_{j} \alpha_{1}\right)\left(c_{j}+d_{j} \alpha_{2}\right)=t_{j} t_{j}^{v} \in \mathcal{O}_{v}^{\times}$. Thus $g \in K_{v}$ and $\theta(g)=h$.

Proposition 3.3. If the index of the orbit containing the standard representative $x$ is (in ur) then $\operatorname{vol}\left(K_{v} \cap G_{x k_{v}}^{\circ}\right)=1$. If it is $(\mathrm{rm} \mathrm{rm})^{*}$ then $\operatorname{vol}\left(K_{v} \cap G_{x k_{v}}^{\circ}\right)=\left(1-q_{v}^{-1}\right)^{-1} q_{v}^{-\tilde{\delta}_{v}}$.

Proof. For these cases $H_{x \mathcal{O}_{v}}=\left(\tilde{\mathcal{O}}_{v}^{\times}\right)^{2}$ and $\theta$ is $\theta\left(A_{p}(c, d), *\right)=\left(c+d \alpha_{1}, c+d \alpha_{2}\right)$. Note that we get this formula by explicitly computing $g_{p} s_{x}\left(t_{x}\right) g_{p}^{-1}$ for $t_{x}=\left(c+d \alpha_{1}\right.$, $\left.c+d \alpha_{2}\right)$ in (2.26). Given $t_{1}, t_{2} \in \tilde{\mathcal{O}}_{v}^{\times}$we wish to determine whether there are integers $c, d \in \tilde{\mathcal{O}}_{v}$ such that $\theta\left(A_{p}(c, d), *\right)=\left(t_{1}, t_{2}\right)$, because $\left(t_{1}, t_{2}\right) \in \theta\left(K_{v} \cap G_{x k_{v}}^{\circ}\right)$ if and only if this is possible. Elementary linear algebra shows that

$$
c=\left(\alpha_{2} t_{1}-\alpha_{1} t_{2}\right) /\left(\alpha_{2}-\alpha_{1}\right), \quad d=\left(t_{2}-t_{1}\right) /\left(\alpha_{2}-\alpha_{1}\right) .
$$

Thus $\left(t_{1}, t_{2}\right) \in \theta\left(K_{v} \cap G_{x k_{v}}^{\circ}\right)$ if and only if $\alpha_{2}-\alpha_{1}$ divides $t_{2}-t_{1}$ and $\alpha_{2} t_{1}-\alpha_{1} t_{2}$. If $\tilde{k}_{v}=k_{v}(x)$ is unramified over $k_{v}$ then $\alpha_{2}-\alpha_{1}$ is a unit and this is no condition on $t_{1}$ and $t_{2}$. Thus $\theta\left(K_{v} \cap G_{x k_{v}}^{\circ}\right)=H_{x \mathfrak{O}_{v}}$ in this case and the first claim follows.

If $\tilde{k}_{v}=k_{v}(x)$ is ramified over $k_{v}$ then $\left(\alpha_{2}-\alpha_{1}\right)=\tilde{\mathfrak{p}}_{v}^{\tilde{\delta}_{v}}$ and so we must have $t_{1} \equiv t_{2}\left(\tilde{\mathfrak{p}}_{v}^{\tilde{\delta}_{v}}\right) . \quad$ With this condition

$$
\alpha_{2} t_{1}-\alpha_{1} t_{2}=\left(\alpha_{2}-\alpha_{1}\right) t_{1}+\alpha_{1}\left(t_{1}-t_{2}\right) \in \tilde{\mathfrak{p}}_{v}
$$

and so the second condition also holds. Thus

$$
\begin{aligned}
\theta\left(K_{v} \cap G_{x k_{v}}^{\circ}\right) & =\left\{\left(t_{1}, t_{2}\right) \in H_{x \mathcal{O}_{v}} \mid t_{1} \equiv t_{2}\left(\tilde{\mathfrak{p}}_{v}^{\tilde{\delta}_{v}}\right)\right\} \\
& \cong\left\{\left(\bar{t}_{1}, \bar{t}_{2}\right) \in H_{x \mathcal{O}_{v}} \mid \bar{t}_{1} \equiv 1\left(\tilde{\mathfrak{p}}_{v}^{\tilde{\delta}_{v}}\right)\right\}
\end{aligned}
$$


where the isomorphism is by the measure preserving map $\left(t_{1}, t_{2}\right) \mapsto\left(t_{1} t_{2}^{-1}, t_{2}\right)$. Thus $\operatorname{vol}\left(K_{v} \cap G_{x k_{v}}^{\circ}\right)=\operatorname{vol}\left(1+\tilde{\mathfrak{p}}_{v}^{\tilde{\delta}_{v}}\right)$ under the normalized multiplicative Haar measure on $\tilde{k}_{v}^{\times}$. The second claim follows.

In the remaining cases, the fields $\tilde{k}_{v}$ and $k_{v}(x)$ are distinct quadratic extensions of $k_{v}$ and we shall assume this to be so for the rest of this section.

LEMMA 3.4. We have $\theta\left(K_{v} \cap G_{x k_{v}}^{\circ}\right)=\tilde{\mathscr{O}}_{v}\left[\alpha_{1}\right]^{\times}$.

ProOF. As pointed out in the proof of Lemma 3.30 in [2] (whose statement is recalled in Lemma 2.15 here), the map $\theta$ is $\theta\left(A_{p}(c, d), *\right)=c+d \alpha_{1} \in \tilde{k}_{v}(x)^{\times}$. This can also easily be verified by explicit computation. If $\kappa \in K_{v} \cap G_{x k_{v}}^{\circ}$ and $\kappa=\left(A_{p}(c, d), *\right)$ then $c, d \in \tilde{\mathcal{O}}_{v}$ and so $\theta(\kappa) \in \tilde{\mathcal{O}}_{v}\left[\alpha_{1}\right]$. Moreover, $\operatorname{det} A_{p}(c, d)=\theta(\kappa) \theta(\kappa)^{v}$ and since $\operatorname{det} A_{p}(c, d) \in \tilde{\mathcal{O}}_{v}^{\times}$and $\tilde{\mathcal{O}}_{v}\left[\alpha_{1}\right]$ is stable under $v$, it follows that $\theta(\kappa)$ is a unit in the ring $\tilde{\mathcal{O}}_{v}\left[\alpha_{1}\right]$. Thus $\theta\left(K_{v} \cap G_{x k_{v}}^{\circ}\right) \subseteq \tilde{\mathcal{O}}_{v}\left[\alpha_{1}\right]^{\times}$. Suppose that $c+d \alpha \in \tilde{\mathcal{O}}_{v}\left[\alpha_{1}\right]^{\times}$. Then $c, d \in \tilde{\mathcal{O}}_{v}$ and $\operatorname{det} A_{p}(c, d)=\left(c+d \alpha_{1}\right)\left(c+d \alpha_{2}\right) \in \tilde{\mathcal{O}}_{v}^{\times}$and it follows from Lemma 3.1 that $\left(A_{p}(c, d), *\right) \in K_{v} \cap G_{x k_{v}}^{\circ}$. This establishes the reverse inclusion.

Proposition 3.5. If the index of the orbit containing the standard representative $x$ is ( $\mathrm{rm}$ ur) or (in $\mathrm{rm})$ then $\operatorname{vol}\left(K_{v} \cap G_{x k_{v}}^{\circ}\right)=1$.

Proof. If the index is (rm ur) then $p(z) \in k_{v}[z]$ either has the form $p(z)=z^{2}-r$ with $r$ a non-square unit, if $v \notin \mathfrak{M}_{\mathrm{dy}}$, or is an Artin-Schreier polynomial, if $v \in \mathfrak{M}_{\mathrm{dy}}$. By hypothesis, $p(z)$ is irreducible when regarded as an element of $\tilde{k}_{v}[z]$. Thinking of $\tilde{k}_{v}$ as the ground field, these facts imply that $\mathcal{O}_{\tilde{k}_{v}(x)}=\tilde{\mathcal{O}}_{v}\left[\alpha_{1}\right]$ and so $\theta\left(K_{v} \cap G_{x k_{v}}^{\circ}\right)=\mathcal{O}_{\tilde{k}_{v}(x)}^{\times}=$ $H_{x \mathcal{U}_{v}}$, by Lemma 3.4.

If the index is (in $\mathrm{rm}$ ) then $p(z) \in k_{v}[z]$ is an Eisenstein polynomial and, since $\tilde{k}_{v} / k_{v}$ is unramified, $p(z)$ is still an Eisenstein polynomial when regarded as an element of $\tilde{k}_{v}[z]$. Thus $\mathcal{O}_{\tilde{k}_{v}(x)}=\tilde{\mathcal{O}}_{v}\left[\alpha_{1}\right]$ and, again by the Lemma, $\theta\left(K_{v} \cap G_{x k_{v}}^{\circ}\right)=H_{x \mathcal{O}_{v}}$.

We are left with the index (rm rm ur) with $v \notin \mathfrak{M}_{\text {dy }}$. In this case $\tilde{k}_{v}(x)=$ $k_{v}\left(\sqrt{\pi_{v}}, \sqrt{\eta}\right)$ where $\pi_{v}$ is a uniformizer and $\eta$ is a non-square unit. We may assume without loss of generality that $\tilde{k}_{v}=k_{v}\left(\sqrt{\pi_{v}}\right)$ and $k_{v}(x)=k_{v}\left(\sqrt{\pi_{v} \eta}\right)$.

Proposition 3.6. If $v \in \mathfrak{M}_{\mathrm{f}} \backslash \mathfrak{M}_{\mathrm{dy}}$ and $x$ is the standard orbital representative for an orbit with index ( $\mathrm{rm}$ rm ur) then $\operatorname{vol}\left(K_{v} \cap G_{x k_{v}}^{\circ}\right)=\left(q_{v}+1\right)^{-1}$.

Proof. We may choose $p(z)=z^{2}-\pi_{v} \eta$ as the Eisenstein polynomial associated to the extension $k_{v}(x) / k_{v}$. Then $\alpha_{1}=\sqrt{\pi_{v} \eta}$ and so $\theta\left(K_{v} \cap G_{x k_{v}}^{\circ}\right)=\tilde{\mathcal{O}}_{v}\left[\sqrt{\pi_{v} \eta}\right]^{\times}$by Lemma 3.4. Since $\tilde{\mathcal{O}}_{v}=\mathcal{O}_{v}\left[\sqrt{\pi_{v}}\right]$, we have

$$
\tilde{\mathcal{O}}_{v}\left[\sqrt{\pi_{v} \eta}\right]^{\times}=\left\{e_{1}+e_{2} \pi_{v} \sqrt{\eta}+e_{3} \sqrt{\pi_{v}}+e_{4} \sqrt{\pi_{v} \eta} \mid e_{1} \in \mathcal{O}_{v}^{\times}, e_{2}, e_{3}, e_{4} \in \mathcal{O}_{v}\right\} .
$$

On the other hand, $\mathcal{O}_{\tilde{k}_{v}(x)}=\tilde{\mathcal{O}}_{v}[\sqrt{\eta}]$ and so $H_{x \mathcal{O}_{v}}=\mathcal{O}_{\tilde{k}_{v}(x)}^{\times}$is

$$
\left\{f_{1}+f_{2} \sqrt{\eta}+f_{3} \sqrt{\pi_{v}}+f_{4} \sqrt{\pi_{v} \eta} \mid f_{1}, \ldots, f_{4} \in \mathcal{O}_{v}, f_{1} \text { or } f_{2} \text { a unit }\right\} .
$$

From this it is clear that

$$
\left[H_{x \mathcal{O}_{v}}: \theta\left(K_{v} \cap G_{x k_{v}}^{\circ}\right)\right]=q_{v}+1
$$

and so the volume has the indicated value. 


\section{Orbital volumes at the finite places.}

In this section we compute $\operatorname{vol}\left(K_{v} x\right)$ for all standard orbital representatives, $x$, when $v$ is a finite, non-dyadic place. When $v$ is dyadic we compute the sum of $\operatorname{vol}\left(K_{v} x\right)$ over the equivalence class of $x$ under the relation $\asymp$ introduced in Section 2, unless $x$ has type $(\mathrm{rm} \mathrm{rm})^{*},(\mathrm{rm} \mathrm{rm} \mathrm{ur})$, or $(\mathrm{rm} \mathrm{rm} \mathrm{rm})$. These cases will be treated in [3]. Throughout this section, $v$ will be a finite place of $k$.

Our strategy is to find a subset $\mathscr{D}$ of $K_{v} x$, defined by congruence conditions, whose translates cover the orbit and then to find its stabilizer modulo a certain high power of the prime ideal. Since the entries of elements of $V$ may lie in different fields we need a notation for congruences that takes this into account. Suppose that $v \notin \mathfrak{M}_{\mathrm{sp}}$. We use the coordinate system (2.21) on $V_{k_{v}}$. If $x=\left(x_{i j}\right)$ and $y=\left(y_{i j}\right)$ are written with respect to this coordinate system then $x \equiv y\left(\mathfrak{p}_{v}^{n_{1}}, \tilde{\mathfrak{p}}_{v}^{n_{2}}\right)$ means that $x_{i 0} \equiv y_{i 0}\left(\mathfrak{p}_{v}^{n_{1}}\right), x_{i 2} \equiv y_{i 2}\left(\mathfrak{p}_{v}^{n_{1}}\right)$ and $x_{i 1} \equiv y_{i 1}\left(\tilde{\mathfrak{p}}_{v}^{n_{2}}\right)$ for $i=1,2$. If $v \in \mathfrak{M}_{\mathrm{sp}}$ then $x \equiv y\left(\mathfrak{p}_{v}^{n}\right)$ will signify congruences to the modulus $\mathfrak{p}_{v}^{n}$ on all the corresponding entries of the two pairs of matrices.

In addition to this, we shall require some further notation as follows: We put $2 \mathcal{O}_{v}=\mathfrak{p}_{v}^{m_{v}}$ (so that $m_{v}=0$ unless $v$ is dyadic) and if $k_{v}(x) / k_{v}$ is quadratic then we put $\Delta_{k_{v}(x) / k_{v}}=\mathfrak{p}_{v}^{\delta_{x, v}}$. If $p(z)=z^{2}+a_{1} z+a_{2} \in k_{v}[z]$ then $\alpha=\left\{\alpha_{1}, \alpha_{2}\right\}$ will be its set of roots. Our standard orbital representative for orbits with $k_{v}(x)=k_{v}$ is $x=w_{p}$ with $p(z)=z^{2}-z$. However, we shall find it convenient to make use of $x=w$ instead. Note that if $g_{p}$ is as in (2.10) then $g_{p} \in K_{v}$ and so $K_{v} w_{p}=K_{v} w$ and this substitution is permissible. So we shall use $w$ as the orbital representative for its orbit.

Since $w, w_{p} \in G_{\mathfrak{O}_{v}}, G_{w}$ and $G_{w_{p}}$ are defined over $\mathcal{O}_{v}$. So for any $j \geq 0$ we may consider $G_{x \mathcal{O}_{v} / \mathfrak{p}_{v}^{j+1}}$ and $G_{x \mathcal{V}_{v} / \mathfrak{p}_{v}^{j+1}}^{\circ}$. We may also consider the reduction $\bar{x}$ of $x$ modulo $\mathfrak{p}_{v}^{j+1}$ and its stabilizer, $G_{\bar{x} \mathscr{v}_{v} / \mathfrak{p}_{v}^{j+1}}$. In general, $G_{x \mathscr{Q}_{v} / \mathfrak{p}_{v}^{j+1}}$ and $G_{\bar{x}_{v} / \mathfrak{p}_{v}^{j+1}}$ may not coincide and we have to proceed carefully. However, $G_{x \hat{Q}_{v} / \mathfrak{p}_{v}^{j+1}} \subseteq G_{\bar{x}_{v} / \mathfrak{p}_{v}^{j+1}}$ in all cases.

The following lemma describes $G_{x \mathfrak{O}_{v} / \mathfrak{p}_{v}^{j+1}}$, when $v \in \mathfrak{M}_{\text {sp. }}$.

Lemma 4.1. Suppose $v \in \mathfrak{M}_{\mathrm{sp}}$ and $x=w_{p}$ is a standard orbital representative. Then $G_{x \mathcal{O}_{v} / \mathfrak{p}_{v}^{j+1}}^{\circ}$ consists of elements of the form (2.13) such that

$$
\operatorname{det} A_{p}\left(c_{1}, d_{1}\right), \operatorname{det} A_{p}\left(c_{2}, d_{2}\right) \in\left(\mathcal{O}_{v} / \mathfrak{p}_{v}^{j+1}\right)^{\times}
$$

and $c_{3}, d_{3} \in \mathcal{O}_{v} / \mathfrak{p}_{v}^{j+1}$ are related to $c_{1}, c_{2}, d_{1}, d_{2} \in \mathcal{O}_{v} / \mathfrak{p}_{v}^{j+1}$ by (2.14). Moreover,

$$
\left[G_{x \mathcal{O}_{v} / \mathfrak{p}_{v}^{j+1}}: G_{x \mathcal{O}_{v} / \mathfrak{p}_{v}^{j+1}}^{\circ}\right]=2
$$

and the non-trivial class in $G_{x \mathfrak{Q}_{v} / \mathfrak{p}_{v}^{j+1}} / G_{x \mathfrak{Q}_{v} / \mathfrak{p}_{v}^{j+1}}^{\circ}$ is represented by $\left(\tau_{p}, \tau_{p}, \tau_{p}\right)$.

Proof. We briefly sketch the proof here. The conditions in Lemma 2.12 determine the structure of $G_{x}$ as a scheme over $\mathcal{O}_{v}$ regarding $c_{1}, d_{1}, c_{2}, d_{2}$ as variables. Note that the inequalities $A_{p}\left(c_{1}, d_{1}\right), A_{p}\left(c_{2}, d_{2}\right) \neq 0$ can be regarded as equations $u_{1} A_{p}\left(c_{1}, d_{1}\right)=u_{2} A_{p}\left(c_{2}, d_{2}\right)=1$ after adding variables $u_{1}, u_{2}$. Then $G_{x \mathcal{O}_{v} / p_{v}^{j+1}}$ is by definition the set of $\left(\mathcal{O}_{v} / \mathfrak{p}_{v}^{j+1}\right)$-valued points of this scheme and so the equation (2.14) will be regarded as an equation over $\mathcal{O}_{v} / \mathfrak{p}_{v}^{j+1}$ and the above inequalities, after reduction modulo $\mathfrak{p}_{v}^{j+1}$, can be regarded as the condition that $A_{p}\left(c_{1}, d_{1}\right), A_{p}\left(c_{2}, d_{2}\right)$ are units. Since $\left(\tau_{p}, \tau_{p}, \tau_{p}\right)$ is defined over $\mathcal{O}_{v}$, this proves the lemma. 
Similarly, the following is an easy consequence of Lemma 2.15 and we will not give a proof.

Lemma 4.2. Suppose $v \notin \mathfrak{M}_{\mathrm{sp}}$ and $x=w_{p}$ is a standard orbital representative. Then $G_{x \mathcal{Q}_{v} / \mathfrak{p}_{v}^{j+1}}^{\circ}$ consists of elements of the form (2.16) such that $\operatorname{det} A_{p}\left(c_{1}, d_{1}\right) \in\left(\tilde{\mathcal{O}}_{v} / \tilde{\mathfrak{p}}_{v}^{j+1}\right)^{\times}$or $\left(\tilde{\mathcal{O}}_{v} / \tilde{\mathfrak{p}}_{v}^{2(j+1)}\right)^{\times}$, according as $\tilde{k}_{v} / k_{v}$ is ramified or unramified, and $c_{2}, d_{2} \in \mathcal{O}_{v} / \mathfrak{p}_{v}^{j+1}$ are related to $c_{1}, d_{1} \in \tilde{\mathcal{O}}_{v} / \tilde{\mathfrak{p}}_{v}^{j+1}$ or $\tilde{\mathcal{O}}_{v} / \tilde{\mathfrak{p}}_{v}^{2(j+1)}$ by (2.17). Moreover,

$$
\left[G_{x \mathcal{U}_{v} / \mathfrak{p}_{v}^{j+1}}: G_{x \mathcal{U}_{v} / \mathfrak{p}_{v}^{j+1}}^{\circ}\right]=2
$$

and the non-trivial class in $G_{x \mathfrak{Q}_{v} / \mathfrak{p}_{v}^{j+1}} / G_{x \mathfrak{Q}_{v} / \mathfrak{p}_{v}^{j+1}}^{\circ}$ is represented by $\left(\tau_{p}, \tau_{p}\right)$.

Definition 4.3. For $x=\left(x_{1}, x_{2}\right) \in V_{\mathcal{O}_{v} / \mathfrak{p}_{v}^{j+1}}$ let $\operatorname{Span}(x)$ be the $\mathcal{O}_{v} / \mathfrak{p}_{v}^{j+1}$-module generated by $x_{1}$ and $x_{2}$.

The following simple observation will be useful below. We will usually apply it with $x$ being the reduction modulo $\mathfrak{p}_{v}^{j+1}$ of a standard orbital representative.

LeMma 4.4. Let $v \in \mathfrak{M}_{\mathrm{sp}}$. Given $g_{1}, g_{2} \in G L(2)_{\mathcal{O}_{v} / \mathfrak{p}_{v}^{j+1}}$, there exists an element $g_{3} \in$ $G L(2)_{\mathcal{O}_{v} / \mathfrak{p}_{v}^{j+1}}$ such that $\left(g_{1}, g_{2}, g_{3}\right) \in G_{x \mathcal{O}_{v} / \mathfrak{p}_{v}^{j+1}}$ if and only if $\operatorname{Span}\left(\left(g_{1}, g_{2}, 1\right) x\right)=\operatorname{Span}(x)$. Similarly, let $v \notin \mathfrak{M}_{\mathrm{sp}}$. Given $g_{1} \in G L(2)_{\tilde{\mathfrak{U}}_{v} / \tilde{\mathfrak{p}}_{v}^{j+1}}$ or $G L(2)_{\tilde{\mathfrak{U}}_{v} / \tilde{\mathfrak{p}}_{v}^{2(j+1)}}$, according as $v \in \mathfrak{M}_{\text {in }}$ or $v \in \mathfrak{M}_{\mathrm{rm}}$, there exists $g_{2} \in G L(2)_{\mathfrak{O}_{v} / \mathfrak{p}_{v}^{j+1}}$ such that $\left(g_{1}, g_{2}\right) \in G_{x \mathfrak{O}_{v} / \mathfrak{p}_{v}^{j+1}}$ if and only if $\operatorname{Span}\left(\left(g_{1}, 1\right) x\right)=\operatorname{Span}(x)$.

Before continuing, we would like to mention a simple fact which we shall have to use frequently below. If $v$ is a finite, non-dyadic place of $k$ and $v \notin \mathfrak{M}_{\text {sp }}$ then any class in $\tilde{\mathcal{O}}_{v} / \tilde{\mathfrak{p}}_{v}^{j}$ which is fixed by $\sigma$ has a representative which lies in $\mathcal{O}_{v}$. To see this, simply observe that if $u \in \tilde{\mathcal{O}}_{v}$ represents such a class then $u^{\sigma} \equiv u\left(\tilde{\mathfrak{p}}_{v}^{j}\right)$ and so $u^{\prime}=\left(u+u^{\sigma}\right) / 2$ satisfies $u^{\prime} \equiv u\left(\tilde{\mathfrak{p}}_{v}^{j}\right)$ and $u^{\prime} \in \mathcal{O}_{v}$. Of course, the corresponding claim when $v$ is a dyadic place is false.

PROPOSITION 4.5. If $k_{v}(x) / k_{v}$ is not ramified then $G_{\bar{x} \mathcal{G}_{v} / \mathfrak{p}_{v}^{j+1}}=G_{x \mathcal{Q}_{v} / \mathfrak{p}_{v}^{j+1}}$.

Proof. We first consider the case $x=w$. Suppose $v \in \mathfrak{M}_{\mathrm{sp}}$. Note that $G_{w \mathcal{O}_{v} / \mathfrak{p}_{v}^{j+1}} \cong$ $\left(\left(\mathcal{O}_{v} / \mathfrak{p}_{v}^{j+1}\right)^{\times}\right)^{4}$ in this case. Let $g=\left(g_{1}, g_{2}, g_{3}\right) \in G_{\bar{w} \mathcal{O}_{v} / \mathfrak{p}_{v}^{j+1}}$ be as in (2.19) where the entries are elements of $\mathcal{O}_{v} / \mathfrak{p}_{v}^{j+1}$. By computation,

$$
\left(g_{1}, g_{2}, 1\right) w=\left(\left(\begin{array}{ll}
g_{111} g_{211} & g_{111} g_{221} \\
g_{121} g_{211} & g_{121} g_{221}
\end{array}\right),\left(\begin{array}{ll}
g_{112} g_{212} & g_{112} g_{222} \\
g_{122} g_{212} & g_{122} g_{222}
\end{array}\right)\right)
$$

Since $\operatorname{Span}\left(\left(g_{1}, g_{2}, 1\right) \bar{w}\right)=\operatorname{Span}(\bar{w})$,

$$
g_{111} g_{221}=g_{121} g_{211}=g_{112} g_{222}=g_{122} g_{212}=0 .
$$

If $g_{111}$ is a unit then $g_{221}=0$. Thus $g_{211}, g_{222}$ are units. So $g_{112}=g_{121}=0$. This implies $g_{122}$ is a unit and so $g_{212}=0$. If $g_{111}$ is not a unit, $g_{121}$ must be a unit. By a similar argument, we can conclude that $g_{111}=g_{122}=g_{211}=g_{222}=0$ and $g_{112}, g_{121}, g_{212}$, $g_{221}$ are units. Multiplying by an element of $G_{x \mathcal{O}_{v} / p_{v}^{j+1}}$ if necessary, we may assume that $g_{1}=g_{2}=1$. Then it is easy to see that $g_{3}=1$. It follows that $G_{\bar{w} \mathcal{O}_{v} / \mathfrak{p}_{v}^{j+1}}=G_{w \mathcal{w}_{v} / \mathfrak{p}_{v}^{j+1}}$. The case $v \notin \mathfrak{M}_{\mathrm{sp}}$ is similar. 
We now assume $k_{v}(x) / k_{v}$ is quadratic and unramified. Suppose $v \in \mathfrak{M}_{\mathrm{sp}}$. Let $g=$ $\left(g_{1}, g_{2}, g_{3}\right) \in G_{\bar{x} \hat{O}_{v} / \mathfrak{p}_{v}^{j+1}}$. We choose representatives of $g_{1}, g_{2}, g_{3}$ in $G L(2)_{\mathcal{O}_{v}}$ and use the same notation. Since $k_{v}(x) / k_{v}$ is unramified, if $c, d \in \mathcal{O}_{v}, c+d \alpha_{1}$ is a unit if and only if either $c$ or $d$ is a unit. The $(1,1)$-entry of $A_{p}\left(c_{i}, d_{i}\right) g_{i}$ is $c_{i} g_{i 11}-d_{i} g_{i 21}$ and the $(1,2)$ entry is $c_{i} g_{i 12}-d_{i} g_{i 22}$. Since either $g_{i 12}$ or $g_{i 22}$ is a unit, we may choose $c_{i}=g_{i 22}$ and $d_{i}=g_{i 12}$ to make the $(1,2)$-entry of $A_{p}\left(c_{i}, d_{i}\right) g_{i}$ zero. Replacing $g_{i}$ by an element of the form $\left(A_{p}\left(c_{1}, d_{1}\right), A_{p}\left(c_{2}, d_{2}\right), A_{p}\left(c_{3}, d_{3}\right)\right) g$ (with $c_{3}, d_{3}$ determined by $c_{1}, c_{2}, d_{1}, d_{2}$ ), we may assume that

$$
g_{1}=\left(\begin{array}{cc}
1 & 0 \\
u_{1} & t_{1}
\end{array}\right), \quad g_{2}=\left(\begin{array}{cc}
1 & 0 \\
u_{2} & t_{2}
\end{array}\right)
$$

If $x=w_{p}$ and $y=\left(\begin{array}{cc}y_{0} & y_{1} \\ y_{1}^{\sigma} & y_{2}\end{array}\right), \bar{y} \in \operatorname{Span}(\bar{x})$ if and only if $y_{1}=y_{1}^{\sigma}$ and $y_{2}-a_{1} y_{1}+a_{2} y_{0}=0$. By computation, $\left(g_{1}, g_{2}, 1\right) x=\left(M_{1}, M_{2}\right)$ where

$$
\begin{aligned}
M_{1} & =\left(\begin{array}{cc}
0 & t_{2} \\
t_{1} & a_{1} t_{1} t_{2}+t_{2} u_{1}+t_{1} u_{2}
\end{array}\right), \\
M_{2} & =\left(\begin{array}{cc}
1 & a_{1} t_{2}+u_{2} \\
a_{1} t_{1}+u_{1} & u_{1} u_{2}+a_{1}\left(t_{2} u_{1}+t_{1} u_{2}\right)+\left(a_{1}^{2}-a_{2}\right) t_{1} t_{2}
\end{array}\right) .
\end{aligned}
$$

Therefore $t_{1}=t_{2}, u_{1}=u_{2}$, and

$$
a_{1}\left(t_{1}-1\right)+2 u_{1}=0, \quad u_{1}^{2}+a_{1}\left(2 t_{1}-1\right) u_{1}+a_{1}^{2}\left(t_{1}^{2}-t_{1}\right)+a_{2}\left(1-t_{1}^{2}\right)=0 .
$$

If $v$ is not dyadic, $a_{1}=0$. So $u_{1}=t_{1}^{2}-1=0$. Then $g_{1}, g_{2}$ are both the identity matrix or both $-\tau_{p}$. If $v$ is dyadic, $p$ is an Artin-Schreier polynomial. This means $a_{1}=-1$ and $a_{2}$ is a unit. By the first condition of (4.8), $t_{1}=2 u_{1}+1$. Substituting in the second condition and simplifying, $\left(4 a_{2}-1\right)\left(u_{1}^{2}+u_{1}\right)=0$. Since $4 a_{2}-1$ is a unit, $u_{1}^{2}+u_{1}=0$. If $u_{1}$ is a unit, $u_{1}=-1$, and if $u_{1}$ is not a unit, $u_{1}=0$. Note that this argument is valid in $\mathcal{O}_{v} / \mathfrak{p}_{v}^{j+1}$ (even though it is not a field). If $u_{1}=-1$ then $t_{1}=-1$ and $g_{1}, g_{2}$ are both $-\tau_{p}$. If $u_{1}=0$ then $t_{1}=1$ and $g_{1}, g_{2}=1$. Then since $(-1,-1,1)$, $\left(\tau_{p}, \tau_{p}, \tau_{p}\right) \in G_{x \mathcal{Q}_{v} / \mathfrak{p}_{v}^{j+1}}, \quad\left(-\tau_{p},-\tau_{p}, \tau_{p}\right) \in G_{x \mathcal{U}_{v} / \mathfrak{p}_{v}^{j+1}}$. This proves the proposition when $v \in \mathfrak{M}_{\mathrm{sp}}$

Suppose now that $v \notin \mathfrak{M}_{\mathrm{sp}}$. Let $g=\left(g_{1}, g_{2}\right) \in G_{\bar{x}_{v} / \mathfrak{p}_{v}^{j+1}}$ be as in (2.19) where entries of $g_{2}$ are in $\mathcal{O}_{v} / \mathfrak{p}_{v}^{j+1}$ and entries of $g_{1}$ are in $\tilde{\mathcal{O}}_{v} / \tilde{\mathfrak{p}}_{v}^{j+1}$ or $\tilde{\mathcal{O}}_{v} / \tilde{\mathfrak{p}}_{v}^{2(j+1)}$, according as $v \in \mathfrak{M}_{\text {in }}$ or $\mathfrak{M}_{\mathrm{rm}}$. We first show that the right $G_{x \mathcal{O}_{v} / \mathfrak{p}_{v}^{j+1}}$-coset of $g$ contains an element of the form

$$
\left(\left(\begin{array}{cc}
1 & 0 \\
u_{1} & t_{1}
\end{array}\right), *\right)
$$

If $v \in \mathfrak{M}_{\mathrm{rm}}$, roots of $p(z)$ still generates the unique unramified quadratic extension of $\tilde{k}_{v}$ and so an element $c_{1}+d_{1} \alpha_{1}$ with $c_{1}, d_{1} \in \tilde{\mathcal{O}}_{v}$ is a unit if and only if $c_{1}$ or $d_{1}$ is a unit. We choose $c_{1}=g_{122}, d_{1}=g_{112}$. Then either $c_{1}$ or $d_{1}$ is a unit. So there exist $c_{2}, d_{2} \in \mathcal{O}_{v}$ such that $\left(A_{p}\left(c_{1}, d_{1}\right), A_{p}\left(c_{2}, d_{2}\right)\right) \in G_{x \mathcal{O}_{v} / \mathfrak{p}_{v}^{j+1}}$. Multiplying this element by $g$, we may assume that $g_{112}=0$.

If $v \in \mathfrak{M}_{\text {in }}$ then $k_{v}(x)=\tilde{k}_{v}$ is unramified over $k_{v}$. Note that $p(z)$ is irreducible modulo $\mathfrak{p}_{v}$ by assumption. Therefore, $\bar{x} \in V_{\mathscr{O}_{v} / \mathfrak{p}_{v}}^{\text {ss }}$. Since $\mathcal{O}_{v} / \mathfrak{p}_{v}$ is a perfect field, one can 
use the same argument as in Proposition 2.10, (2) [1], p. 323 to determine the stabilizer $G_{\bar{x} \hat{U}_{v} / \mathfrak{p}_{v}}$. So the proposition is true if $j=0$.

By the previous step, we can multiply $g$ by an element of $G_{x \mathcal{V}_{v} / \mathfrak{p}_{v}}$ to assume that $g_{112}, g_{121} \in \tilde{\mathfrak{p}}_{v}$. Let $c_{1}=g_{122}, d_{1}=g_{112}$. Then $c_{1} \in \tilde{\mathcal{O}}_{v}^{\times}$and $d_{1} \in \tilde{\mathfrak{p}}_{v}$. So $c_{1}-d_{1} \alpha_{1} \equiv$ $c_{1}-d_{1} \alpha_{2} \equiv c_{1}\left(\tilde{\mathfrak{p}}_{v}\right)$. Therefore, there exist $c_{2}, d_{2} \in \mathcal{O}_{v}$ such that $\left(A_{p}\left(c_{1}, d_{1}\right), A_{p}\left(c_{2}, d_{2}\right)\right) \in$ $G_{x \mathcal{O}_{v} / \mathfrak{p}_{v}^{j+1}}$. Multiplying $g$ by this element, we may assume $g_{112}=0$.

In both cases, $g_{112}=0$ and so $g_{111}, g_{122} \in \tilde{\mathcal{O}}_{v}^{\times}$. Multiplying by an element of the form $\left(A_{p}\left(c_{1}, 0\right), A_{p}\left(c_{2}, d_{2}\right)\right) \in G_{x \cup_{v} / p_{v}^{j+1}}$, we may further assume that $g_{111}=1$.

This done, $\left(g_{1}, 1\right) w_{p}$ is given by $(4.7)$ with $t_{2}, u_{2}$ replaced by $t_{1}^{\sigma}, u_{1}^{\sigma}$. Therefore, by the same argument, $t_{1}=t_{1}^{\sigma}, u_{1}^{\sigma}=u_{1}$, and the condition (4.8) holds also. After this, the argument is the same as in the case $v \in \mathfrak{M}_{\mathrm{sp}}$.

DeFINITION 4.10. Let $x$ denote any of the standard orbital representatives. In each of the cases enumerated below, we define $\mathscr{D}$ to be the set of $y \in V_{\mathcal{O}_{v}}$ such that $y \equiv x\left(\mathfrak{p}_{v}^{n_{1}}, \tilde{\mathfrak{p}}_{v}^{n_{2}}\right)$ if $v \notin \mathfrak{M}_{\mathrm{sp}}$ or $y \equiv x\left(\mathfrak{p}_{v}^{n}\right)$ if $v \in \mathfrak{M}_{\mathrm{sp}}$, where $n_{1}, n_{2}$ and $n$ are as indicated.

(1) $n=2 m_{v}+1$ if $x$ has type (sp) or (sp ur),

(2) $n=2$ if $x$ has type (sp rm) and $v \notin \mathfrak{M}_{\mathrm{dy}}$,

(3) $n_{1}=n_{2}=2 m_{v}+1$ if $x$ has type (in) or (in ur),

(4) $n_{1}=n_{2}=2$ if $x$ has type (in $\mathrm{rm}$ ) and $v \notin \mathfrak{M}_{\mathrm{dy}}$,

(5) $n_{1}=2 m_{v}+1, n_{2}=4 m_{v}+2$ if $x$ has type (rm) or (rm ur),

(6) $n_{1}=2, n_{2}=4$ if $x$ has type $(\mathrm{rm} \mathrm{rm})^{*}$ or $(\mathrm{rm} \mathrm{rm} \mathrm{ur})$ and $v \notin \mathfrak{M}_{\mathrm{dy}}$.

Proposition 4.11. Let $x$ have one of the types enumerated in Definition 4.10 and $\mathscr{D}$ be the corresponding set. If $y \in \mathscr{D}$ then $k_{v}(y)=k_{v}(x)$.

Proof. By the choice of orbital representatives, $P(x)$ is a unit when $k_{v}(x) / k_{v}$ is not ramified (including the case $\left.k_{v}(x)=k_{v}\right)$ and $(P(x))=\mathfrak{p}_{v}^{\delta_{x, v}}$ when $k_{v}(x) / k_{v}$ is ramified. In cases (1), (3) and (5), we have $P(y) \equiv P(x)\left(\mathfrak{p}_{v}^{2 m_{v}+1}\right)$ and $P(x)$ is a unit. This congruence may be rewritten as $P(y) / P(x) \equiv 1\left(\mathfrak{p}_{v}^{2 m_{v}+1}\right)$. By Hensel's lemma, a unit is a square if and only if it is a square modulo $\mathfrak{p}_{v}^{2 m_{v}+1}$. Note that this is true whether or not $v$ is dyadic. So $P(y) / P(x)$ is a square. This implies that $k_{v}(y)=k_{v}(x)$ in all these cases.

In case (2), the condition implies that $P(y) \equiv P(x)\left(\mathfrak{p}_{v}^{2}\right)$ and hence that we have $P(y) / P(x) \equiv 1\left(p_{v}\right)$, which again implies that $k_{v}(y)=k_{v}(x)$, since $v$ is not dyadic. The same argument works for case (4) because $\tilde{\mathfrak{p}}_{v}^{2} \cap \mathcal{O}_{v}=\mathfrak{p}_{v}^{2}$ in this case.

In case (6), the assumption implies that $P(y) \equiv P(x)\left(\mathfrak{p}_{v}^{2}\right)$ and $\operatorname{ord}_{k_{v}}(P(x))=1$. So $P(y) P(x)^{-1} \equiv 1\left(\mathfrak{p}_{v}\right)$. Since $v$ is not dyadic, $P(y) P(x)^{-1} \in\left(k_{v}^{\times}\right)^{2}$. Therefore, $k_{v}(y)=$ $k_{v}(x)$.

Before proving the next proposition we recall the notion of omega sets. Let $x \in V_{k_{v}}^{\mathrm{ss}}$.

DeFINITION 4.12. A set $\Omega_{x, v} \subseteq G_{k_{v}}$ is called an omega set for $x$ if it has the following properties:

(1) $\Omega_{x, v} x=\left(G_{k_{v}} x\right) \cap V_{\mathcal{O}_{v}}$.

(2) $K_{v} \Omega_{x, v} \theta_{g_{x}}^{-1}\left(H_{x \mathcal{O}_{v}}\right)=\Omega_{x, v}$.

(3) If $g_{1}, g_{2} \in \Omega_{x, v}, h \in G_{x k_{v}}^{\circ}$ and $g_{1}=g_{2} h$ then $h \in \theta_{g_{x}}^{-1}\left(H_{x \mathcal{G}_{v}}\right)$.

(4) If $g \in \Omega_{x, v}$ then $|\chi(g)|_{v} \leq 1$ with equality only if $g \in K_{v}$. 
We have established in Section 8 [2] that omega sets exist for the representatives of all the orbits with the indices considered in this section.

Proposition 4.13. Let $x$ have one of the types enumerated in Definition 4.10 and $\mathscr{D}$ be the corresponding set. Then $\mathscr{D} \subseteq K_{v} x$.

Proof. We first deal with (1)-(5) in Definition 4.10. Let $\Omega_{x, v}$ be the omega set for $x$. Suppose that $y \in \mathscr{D}$. Then $k_{v}(y)=k_{v}(x)$ and so $y \in G_{k_{v}} x$. Since $y$ is also integral, it follows from the first property of omega sets that $y \in \Omega_{x, v} x$. That is, $y=g x$ for some $g \in \Omega_{x, v}$. We have seen above that the congruence conditions on $y$ also imply that $|P(y)|_{v}=|P(x)|_{v}$ and so $g \in\left\{\left.g \in \Omega_{x, v}|| \chi(g)\right|_{v}=1\right\}=K_{v}$ by the last property of omega sets. This proves the claim for $(1)-(5)$.

Consider case (6). Let

$$
G\left(\pi_{v}^{2}\right)=\left\{g \in K_{v} \mid g_{1} \equiv 1\left(\tilde{\mathfrak{p}}_{v}^{4}\right), g_{2} \equiv 1\left(\mathfrak{p}_{v}^{2}\right)\right\} .
$$

Then $G\left(\pi_{v}^{2}\right)$ fixes the set $\mathscr{D}$. Consider the usual coordinates $y=\left(y_{i j}\right)$ as in (2.21). Since $y_{20} \equiv 1\left(\mathfrak{p}_{v}^{2}\right),\left(1, a\left(y_{20}^{-1}, 1\right)\right) \in G\left(\pi_{v}^{2}\right)$. Applying this element, we may assume that $y_{20}=1$. Since $y_{10} \equiv 0\left(\mathfrak{p}_{v}^{2}\right),\left(1,{ }^{t} n\left(-y_{10}\right)\right) \in G\left(\pi_{v}^{2}\right)$. Applying this element, we may assume that $y_{10}=0$. Since $y_{11} \equiv 1\left(\tilde{\mathfrak{p}}_{v}^{4}\right),\left(a\left(y_{11}^{-1}, 1\right), 1\right) \in G\left(\pi_{v}^{2}\right)$. Applying this element, we may assume that $y_{11}=1$. Since we are assuming $v \notin \mathfrak{M}_{\mathrm{dy}}$, we chose $p$ so that $a_{1}=0$. So $y_{12} \equiv 0\left(\mathfrak{p}_{v}^{2}\right)$. So by a similar argument, we may assume $y_{12}=0$. Also $y_{21} \equiv 0\left(\tilde{\mathfrak{p}}_{v}^{4}\right)$ and $y_{22} \equiv-a_{2}\left(\mathfrak{p}_{v}^{2}\right)$. We may assume that $\tilde{k}_{v}=k_{v}\left(\sqrt{\pi_{v}}\right)$. Let $y_{21}=$ $\pi_{v}^{2}\left(c+d \sqrt{\pi_{v}}\right)$ where $c, d \in \mathcal{O}_{v}$. Then $\left(n\left(-d \pi_{v}^{2} \sqrt{\pi_{v}}\right), n\left(-\pi_{v}^{2} c\right)\right) \in G\left(\pi_{v}^{2}\right)$. Note that $\operatorname{Tr}_{k_{v} / k_{v}}\left(-d \pi_{v}^{2} \sqrt{\pi_{v}}\right)=0$. So applying this element, we may assume that $y_{21}=0$. Then $-y_{22} a_{2}^{-1} \equiv 1\left(\mathfrak{p}_{v}\right)$. So $-y_{22} a_{2}^{-1}=t^{2}$ with $t \in \mathcal{O}_{v}^{\times}$by Hensel's lemma. Applying $\left(a\left(1, t^{-1}\right), a(t, 1)\right)$, we get $y_{22}=-a_{2}$.

Proposition 4.14. (1) If $x$ has type (sp) then $\operatorname{vol}\left(K_{v} x\right)=(1 / 2)\left(1+q_{v}^{-1}\right)\left(1-q_{v}^{-2}\right)^{2}$.

(2) If $x$ has type (sp ur) then $\operatorname{vol}\left(K_{v} x\right)=(1 / 2)\left(1-q_{v}^{-1}\right)^{3}\left(1-q_{v}^{-2}\right)$.

(3) If $x$ has type (in) or (in ur) then $\operatorname{vol}\left(K_{v} x\right)=(1 / 2)\left(1-q_{v}^{-1}\right)\left(1-q_{v}^{-4}\right)$.

(4) If $x$ has type $(\mathrm{rm})$ then $\operatorname{vol}\left(K_{v} x\right)=(1 / 2)\left(1-q_{v}^{-2}\right)^{2}$.

(5) If $x$ has type (rm ur) then $\operatorname{vol}\left(K_{v} x\right)=(1 / 2)\left(1-q_{v}^{-1}\right)^{2}\left(1-q_{v}^{-2}\right)$.

Proof. Let $j=2 m_{v}$ in all cases. Then $\operatorname{vol}\left(K_{v} x\right)=\operatorname{vol}(\mathscr{D}) \#\left(G_{\mathcal{O}_{v} / \mathfrak{p}_{v}^{j+1}} / G_{\bar{x} \mathfrak{Q}_{v} / \mathfrak{p}_{v}^{j+1}}\right)$. By Proposition 4.5, $G_{\bar{x} \mathcal{Q}_{v} / \mathfrak{p}_{v}^{j+1}}=G_{x \mathcal{O}_{v} / \mathfrak{p}_{v}^{j+1}}$. Therefore,

Consider (1). In this case, $G_{x \mathcal{O}_{v} / \mathfrak{p}_{v}^{j+1}}^{\circ} \cong\left(\left(\mathcal{O}_{v} / \mathfrak{p}_{v}^{j+1}\right)^{\times}\right)^{4}$. So its order is $\left(\left(q_{v}-1\right) q_{v}^{j}\right)^{4}$.

$$
\operatorname{vol}\left(K_{v} x\right)=q_{v}^{-8(j+1)} \cdot \frac{\left(q_{v}^{2}-q_{v}\right)^{3}\left(q_{v}^{2}-1\right)^{3}\left(q_{v}^{j}\right)^{12}}{2\left(q_{v}-1\right)^{4}\left(q_{v}^{j}\right)^{4}}=\frac{1}{2}\left(1+q_{v}^{-1}\right)\left(1-q_{v}^{-2}\right)^{2} .
$$

Consider (2) and (3). Let $\mathfrak{p}_{x} \subseteq \mathcal{O}_{k_{v}(x)}$ be the prime ideal. In both cases, $G_{x \mathcal{O}_{v} / \mathfrak{p}_{v}^{j+1}} \cong$ $\left(\left(\mathcal{O}_{x} / \mathfrak{p}_{x}^{j+1}\right)^{\times}\right)^{2}$, and $\#\left(\mathcal{O}_{x} / \mathfrak{p}_{x}\right)=q_{v}^{2}$. So its order is $\left(\left(q_{v}^{2}-1\right) q_{v}^{2 j}\right)^{2}$. Therefore, in case (2),

$$
\operatorname{vol}\left(K_{v} x\right)=q_{v}^{-8(j+1)} \cdot \frac{\left(q_{v}^{2}-q_{v}\right)^{3}\left(q_{v}^{2}-1\right)^{3}\left(q_{v}^{j}\right)^{12}}{2\left(q_{v}^{2}-1\right)^{2}\left(q_{v}^{2 j}\right)^{2}}=\frac{1}{2}\left(1-q_{v}^{-1}\right)^{3}\left(1-q_{v}^{-2}\right) .
$$


In case (3),

$$
\begin{aligned}
\operatorname{vol}\left(K_{v} x\right) & =q_{v}^{-8(j+1)} \cdot \frac{\left(q_{v}^{4}-q_{v}^{2}\right)\left(q_{v}^{4}-1\right)\left(q_{v}^{2 j}\right)^{4}\left(q_{v}^{2}-q_{v}\right)\left(q_{v}^{2}-1\right)\left(q_{v}^{j}\right)^{4}}{2\left(q_{v}^{2}-1\right)^{2}\left(q_{v}^{2 j}\right)^{2}} \\
& =\frac{1}{2}\left(1-q_{v}^{-1}\right)\left(1-q_{v}^{-4}\right) .
\end{aligned}
$$

Consider (4). In this case, $G_{x \mathcal{O}_{v} / \mathfrak{p}_{v}^{j+1}}^{\circ} \cong\left(\left(\tilde{\mathcal{O}}_{v} / \tilde{\mathfrak{p}}_{v}^{2(j+1)}\right)^{\times}\right)^{2}$ and $\#\left(\tilde{\mathcal{O}}_{v} / \tilde{\mathfrak{p}}_{v}\right)=q_{v}$. So its order is $\left(\left(q_{v}-1\right) q_{v}^{2 j+1}\right)^{2}$. Therefore,

$$
\operatorname{vol}\left(K_{v} x\right)=q_{v}^{-8(j+1)} \cdot \frac{\left(q_{v}^{2}-q_{v}\right)^{2}\left(q_{v}^{2}-1\right)^{2}\left(q_{v}^{2 j+1}\right)^{4}\left(q_{v}^{j}\right)^{4}}{2\left(q_{v}-1\right)^{2}\left(q_{v}^{2 j+1}\right)^{2}}=\frac{1}{2}\left(1-q_{v}^{-2}\right)^{2} .
$$

Consider (5). Let $\mathfrak{p}_{x} \subseteq \mathcal{O}_{k_{v}(x)}$ be the prime ideal. In this case, $G_{x \mathcal{O}_{v} / \mathfrak{p}_{v}^{j+1}}^{\circ} \cong$ $\left(\mathcal{O}_{x} / \mathfrak{p}_{x}^{2(j+1)}\right)^{\times}$and $\#\left(\mathcal{O}_{x} / \mathfrak{p}_{x}\right)=q_{v}^{2}$. So its order is $\left(q_{v}^{2}-1\right) q_{v}^{2(2 j+1)}$. Therefore,

$$
\operatorname{vol}\left(K_{v} x\right)=q_{v}^{-8(j+1)} \cdot \frac{\left(q_{v}^{2}-q_{v}\right)^{2}\left(q_{v}^{2}-1\right)^{2}\left(q_{v}^{2 j+1}\right)^{4}\left(q_{v}^{j}\right)^{4}}{2\left(q_{v}^{2}-1\right)\left(q_{v}^{2(2 j+1)}\right)}=\frac{1}{2}\left(1-q_{v}^{-1}\right)^{2}\left(1-q_{v}^{-2}\right) .
$$

Proposition 4.15. (1) Suppose that $x$ has type $(\mathrm{sp} \mathrm{rm})$ and that $v \notin \mathfrak{M}_{\mathrm{dy}}$. Then

$$
\operatorname{vol}\left(K_{v} x\right)=\frac{1}{2} q_{v}^{-1}\left(1-q_{v}^{-1}\right)\left(1-q_{v}^{-2}\right)^{3}
$$

(2) Suppose that $x$ has type (in $\mathrm{rm}$ ) and that $v \notin \mathfrak{M}_{\mathrm{dy}}$. Then

$$
\operatorname{vol}\left(K_{v} x\right)=\frac{1}{2} q_{v}^{-1}\left(1-q_{v}^{-1}\right)\left(1-q_{v}^{-2}\right)\left(1-q_{v}^{-4}\right) .
$$

ProOF. We prove that $\left[G_{\bar{x} \mathcal{O}_{v} / \mathfrak{p}_{v}^{2}}: G_{x \mathcal{Q}_{v} / \mathfrak{p}^{2}}^{\circ}\right]=2 q_{v}$. Let $p(z)=z^{2}+a_{1} z+a_{2}$ be the Eisenstein polynomial corresponding to $x$. Since $v$ is not dyadic, we assume $a_{1}=0$.

Consider (1). Elements of the form $\left(A_{p}\left(c_{1}, d_{1}\right), A_{p}\left(c_{2}, d_{2}\right), A_{p}\left(c_{3}, d_{3}\right)\right)$ are in $G_{x \mathfrak{Q}_{v} / \mathfrak{p}_{v}^{2}}^{\circ}$ if and only if $c_{1}^{2}+a_{2} d_{1}^{2}, c_{2}^{2}+a_{2} d_{2}^{2} \in\left(\mathcal{O}_{v} / \mathfrak{p}_{v}^{2}\right)^{\times}$(see Lemma 4.1). Since $a_{2} \in \mathfrak{p}_{v}$, this is equivalent to $c_{1}, c_{2} \in\left(\mathcal{O}_{v} / \mathfrak{p}_{v}^{2}\right)^{\times}$. So the order of $G_{x \mathcal{O}_{v} / \mathfrak{p}_{v}^{2}}^{\circ}$ is $\left(\left(q_{v}-1\right) q_{v}^{3}\right)^{2}$. Suppose $g=$ $\left(g_{1}, g_{2}, g_{3}\right) \in G_{\bar{x} \mathfrak{o}_{v} / \mathfrak{p}_{v}^{2}}$. Since $F_{x}(v)$ reduces to $v_{1}^{2}$ modulo $\mathfrak{p}_{v}, g_{321} \in \mathfrak{p}_{v} / \mathfrak{p}_{v}^{2}$. Let $x=$ $\left(x_{1}, x_{2}\right)$. Then $\left(g_{1}, g_{2}, 1\right) x_{2}$ is a unit scalar multiple of $x_{2}$ modulo $\mathfrak{p}_{v}$. By computation,

$$
\left(g_{1}, g_{2}, 1\right) x_{2} \equiv\left(\begin{array}{ll}
g_{111} g_{211} & g_{111} g_{221} \\
g_{121} g_{212} & g_{121} g_{222}
\end{array}\right)\left(\mathfrak{p}_{v}\right) .
$$

So $g_{111}, g_{211}$ are units. By a similar argument as in the proof of Proposition 4.5, $g_{121}, g_{221} \in \mathfrak{p}_{v} / \mathfrak{p}_{v}^{2}$. This implies that $g_{122}, g_{222}$ are units. Since

$$
A_{p}\left(c_{i}, d_{i}\right) g_{i}=\left(\begin{array}{cc}
* & c_{i} g_{i 12}-d_{i} g_{i 22} \\
* & *
\end{array}\right),
$$

for $i=1,2$, the right coset $G_{x \mathcal{Q}_{v} / \mathfrak{p}_{v}^{2}}^{\circ} g$ contains an element $g=\left(g_{1}, g_{2}, g_{3}\right)$ where $g_{1}, g_{2}$ are in the form (4.6). Moreover, it is easy to see that $t_{1}, t_{2}, u_{1}, u_{2}$ are determined by the coset $G_{x \hat{Q}_{v} / \mathfrak{p}_{v}^{2}}^{\circ} g$. We use Lemma 4.4 to determine the possibilities for $g$. By computation, 


$$
\left(g_{1}, g_{2}, 1\right) x=\left(\left(\begin{array}{cc}
0 & t_{2} \\
t_{1} & t_{1} u_{2}+t_{2} u_{1}
\end{array}\right),\left(\begin{array}{cc}
1 & u_{2} \\
u_{1} & u_{1} u_{2}-a_{2} t_{1} t_{2}
\end{array}\right)\right) .
$$

The condition $\operatorname{Span}\left(\left(g_{1}, g_{2}, 1\right) x\right)=\operatorname{Span}(x)$ is equivalent to the condition that $t_{1}=t_{2}$, $u_{1}=u_{2}, t_{1} u_{2}+t_{2} u_{1}=0$, and $u_{1} u_{2}-a_{2} t_{1} t_{2}=-a_{2}$. Since $v$ is not dyadic, $u_{1}=u_{2}=0$ and $a_{2}\left(t_{1}^{2}-1\right)=0$. Therefore, $t_{1} \equiv \pm 1\left(\mathfrak{p}_{v}\right)$. So there are $2 q_{v}$ possibilities for $t_{1}$ modulo $\mathfrak{p}_{v}^{2}$. This proves that $\left[G_{\bar{x} \hat{\vartheta}_{v} / \mathfrak{p}_{v}^{2}}: G_{x_{v} / \mathfrak{p}_{v}^{2}}^{\circ}\right]=2 q_{v}$.

In case (2), by a similar argument as in case (1), we can assume that $g_{1}$ is in the form (4.6) with $t_{1}$ unit and $u_{1} \in \tilde{\mathcal{O}}_{v} / \tilde{\mathfrak{p}}_{v}^{2}$. Note that the order of $G_{x \mathcal{U}_{v} / \mathfrak{p}_{v}^{2}}^{\circ}$ is $\left(q_{v}^{2}-1\right) q_{v}^{6}$ in this case. By the same consideration, we get $t_{1}=t_{1}^{\sigma}, u_{1}=u_{1}^{\sigma}$, and the rest of the conditions turn out to be the same. Since $\tilde{\mathfrak{p}}_{v}^{2} \cap \mathcal{O}_{v}=\mathfrak{p}_{v}^{2}$, there are $2 q_{v}$ possibilities for $t_{1}$. So $\left[G_{\bar{x} \mathfrak{Q}_{v} / \mathfrak{p}_{v}^{2}}: G_{x \mathcal{Q}_{v} / \mathfrak{p}_{v}^{2}}^{\circ}\right]=2 q_{v}$ in this case also.

Since the volume of $\mathscr{D}$ in Definition 4.10 (2) is $q_{v}^{-16}$, if $v \in \mathfrak{M}_{\mathrm{sp}}$,

$$
\operatorname{vol}\left(K_{v} x\right)=q_{v}^{-16} \cdot \frac{\left(q_{v}^{2}-q_{v}\right)^{3}\left(q_{v}^{2}-1\right)^{3} q_{v}^{12}}{2 q_{v}\left(\left(q_{v}-1\right) q_{v}^{3}\right)^{2}}=\frac{1}{2} q_{v}^{-1}\left(1-q_{v}^{-1}\right)\left(1-q_{v}^{-2}\right)^{3}
$$

and if $v \in \mathfrak{M}_{\text {in }}$,

$$
\begin{aligned}
\operatorname{vol}\left(K_{v} x\right) & =q_{v}^{-16} \cdot \frac{\left(q_{v}^{4}-q_{v}^{2}\right)\left(q_{v}^{4}-1\right) q_{v}^{8}\left(q_{v}^{2}-q_{v}\right)\left(q_{v}^{2}-1\right) q_{v}^{4}}{2 q_{v}\left(q_{v}^{2}-1\right) q_{v}^{6}} \\
& =\frac{1}{2} q_{v}^{-1}\left(1-q_{v}^{-1}\right)\left(1-q_{v}^{-2}\right)\left(1-q_{v}^{-4}\right) .
\end{aligned}
$$

Next we assume that $v$ is dyadic and that $x$ has type (sp rm) or (in $\mathrm{rm}$ ). We shall compute the sum of $\operatorname{vol}\left(K_{v} x\right)$ over the equivalence class of $x$ under the relation $\asymp$. For these orbits, this amounts to summing $\operatorname{vol}\left(K_{v} x\right)$ over all $x$ having a given value of $\delta_{x, v}$. Let $x$ be coordinatized as in $(2.20)$ or $(2.21)$. If $v \in \mathfrak{M}_{\mathrm{sp}}$ then $F_{x}(v)=a_{0}(x) v_{1}^{2}+$ $a_{1}(x) v_{1} v_{2}+a_{2}(x) v_{2}^{2}$ where

$$
\begin{aligned}
& a_{0}(x)=x_{112} x_{121}-x_{111} x_{122}, \\
& a_{1}(x)=x_{112} x_{221}+x_{121} x_{212}-x_{111} x_{222}-x_{122} x_{211}, \\
& a_{2}(x)=x_{212} x_{221}-x_{211} x_{222} .
\end{aligned}
$$

If $v \notin \mathfrak{M}_{\mathrm{sp}}$ then $F_{x}(v)=a_{0}(x) v_{1}^{2}+a_{1}(x) v_{1} v_{2}+a_{2}(x) v_{2}^{2}$ where

$$
\begin{aligned}
& a_{0}(x)=\mathrm{N}_{\tilde{k}_{v} / k_{v}}\left(x_{11}\right)-x_{10} x_{12}, \\
& a_{1}(x)=\operatorname{Tr}_{\tilde{k}_{v} / k_{v}}\left(x_{11} x_{21}^{\sigma}\right)-x_{10} x_{22}-x_{12} x_{20}, \\
& a_{2}(x)=\mathrm{N}_{\tilde{k}_{v} / k_{v}}\left(x_{21}\right)-x_{20} x_{22} .
\end{aligned}
$$

Definition 4.18. (1) If $v \in \mathfrak{M}_{\mathrm{sp}}$ then we define $\mathscr{D}_{\ell}$ for $\ell=1, \ldots, m_{v}+1$ to be the set of $x$ such that

$$
\begin{aligned}
& x_{112}, x_{121}, x_{211} \in \mathcal{O}_{v}^{\times}, \quad x_{122}, x_{212}, x_{221} \in \mathfrak{p}_{v}, \\
& \operatorname{ord}_{k_{v}}\left(x_{222}\right)=1, \quad \operatorname{ord}_{k_{v}}\left(a_{1}(x)\right) \begin{cases}=\ell & \text { if } \ell \leq m_{v}, \\
\geq m_{v}+1 & \text { if } \ell=m_{v}+1 .\end{cases}
\end{aligned}
$$


(2) If $v \in \mathfrak{M}_{\text {in }}$ then we define $\mathscr{D}_{\ell}$ for $\ell=1, \ldots, m_{v}+1$ to be the set of $x$ such that

$$
\begin{aligned}
& x_{11} \in \tilde{\mathcal{O}}_{v}^{\times}, \quad x_{20} \in \mathcal{O}_{v}^{\times}, \quad x_{12} \in \mathfrak{p}_{v}, \quad x_{21} \in \tilde{\mathfrak{p}}_{v}, \\
& \operatorname{ord}_{k_{v}}\left(x_{22}\right)=1, \quad \operatorname{ord}_{k_{v}}\left(a_{1}(x)\right) \begin{cases}=\ell & \text { if } \ell \leq m_{v}, \\
\geq m_{v}+1 & \text { if } \ell=m_{v}+1 .\end{cases}
\end{aligned}
$$

Proposition 4.19. (1) If $v \in \mathfrak{M}_{\mathrm{sp}}$ then

$$
\operatorname{vol}\left(\mathscr{D}_{\ell}\right)= \begin{cases}q_{v}^{-3}\left(1-q_{v}^{-1}\right)^{5} q_{v}^{-\ell} & \text { if } \ell \leq m_{v} \\ q_{v}^{-3}\left(1-q_{v}^{-1}\right)^{4} q_{v}^{-\left(m_{v}+1\right)} & \text { if } \ell=m_{v}+1 .\end{cases}
$$

(2) If $v \in \mathfrak{M}_{\text {in }}$ then

$$
\operatorname{vol}\left(\mathscr{D}_{\ell}\right)= \begin{cases}q_{v}^{-3}\left(1-q_{v}^{-1}\right)^{3}\left(1-q_{v}^{-2}\right) q_{v}^{-\ell} & \text { if } \ell \leq m_{v} \\ q_{v}^{-3}\left(1-q_{v}^{-1}\right)^{2}\left(1-q_{v}^{-2}\right) q_{v}^{-\left(m_{v}+1\right)} & \text { if } \ell=m_{v}+1 .\end{cases}
$$

Proof. Suppose $v \in \mathfrak{M}_{\mathrm{sp}}$. It is easy to see that if $x \in V_{\mathcal{Q}_{v}}$ satisfies all the conditions in Definition 4.18(1) except for the last condition, then $a_{0}(x) \in \mathcal{O}_{v}^{\times}$and $\operatorname{ord}_{k_{v}}\left(a_{2}(x)\right)=1$. Suppose

$$
x_{112} x_{221}+x_{121} x_{212}-x_{122} x_{211}=e_{1} \pi_{v}+\cdots+e_{\ell} \pi_{v}^{\ell}+\cdots,
$$

where $e_{j}$ is a unit or zero for all $j$ (see I.4, Corollary 2 in [5], p. 14). Let $x_{222}=\pi_{v} x_{222}^{\prime}$ where $x_{222}^{\prime} \in \mathcal{O}_{v}^{\times}$. Then by [4.16), $a_{1}(x)$ satisfies the condition in Definition 4.18(1) if and only if $x_{111}$ has an expansion of the form

$$
x_{111}=\left(x_{222}^{\prime}\right)^{-1}\left(e_{1}+\cdots+e_{\ell-1} \pi_{v}^{\ell-2}+f \pi_{v}^{\ell-1}+\cdots\right)
$$

such that $f \not \equiv e_{\ell}\left(\mathfrak{p}_{v}\right)$ if $\ell \leq m_{v}$ (and no condition on $f$ if $\ell=m_{v}+1$ ). If $\ell \leq m_{v}$, the volume of the set of such $x_{111}$ is $\left(q_{v}-1\right) q_{v}^{-\ell}=\left(1-q_{v}^{-1}\right) q_{v}^{-\ell+1}$ if $\ell \leq m_{v}$ and $q_{v}^{-m_{v}}$ if $\ell=m_{v}+1$. The volumes of the sets of $x_{112}, x_{121}, x_{211}$ are $1-q_{v}^{-1}$, the volumes of the sets of $x_{122}, x_{212}, x_{221}$ are $q_{v}^{-1}$, and the volume of the set of $x_{222}$ is $q_{v}^{-1}\left(1-q_{v}^{-1}\right)$. Therefore,

$$
\operatorname{vol}\left(\mathscr{D}_{\ell}\right)=\left(1-q_{v}^{-1}\right)^{3} q_{v}^{-3} q_{v}^{-1}\left(1-q_{v}^{-1}\right) \times \begin{cases}\left(1-q_{v}^{-1}\right) q_{v}^{-\ell+1} & \text { if } \ell \leq m_{v} \\ q_{v}^{-m_{v}} & \text { if } \ell=m_{v}+1\end{cases}
$$

Simplifying, we get (1).

If $v \in \mathfrak{M}_{\text {in }}$, similar considerations apply to $x_{10}$ as to $x_{111}$ in the case $v \in \mathfrak{M}_{\mathrm{sp}}$. The volumes of the sets of $x_{11}, x_{20}$ are $1-q_{v}^{-2}$ and $1-q_{v}^{-1}$, respectively, the volumes of the sets of $x_{12}, x_{21}$ are $q_{v}^{-2}$ and $q_{v}^{-1}$, respectively, and the volume of the set of $x_{22}$ is $q_{v}^{-1}\left(1-q_{v}^{-1}\right)$. Therefore,

$$
\operatorname{vol}\left(\mathscr{D}_{\ell}\right)=\left(1-q_{v}^{-2}\right)\left(1-q_{v}^{-1}\right) q_{v}^{-2} q_{v}^{-1} q_{v}^{-1}\left(1-q_{v}^{-1}\right) \times \begin{cases}\left(1-q_{v}^{-1}\right) q_{v}^{-\ell+1} & \text { if } \ell \leq m_{v}, \\ q_{v}^{-m_{v}} & \text { if } \ell=m_{v}+1 .\end{cases}
$$

Simplifying, we get (2).

We employ coordinates on $G$ as in (2.19). If $v \in \mathfrak{M}_{\mathrm{sp}}$ then we define 


$$
\begin{aligned}
& H_{1}=\left\{g \in K_{v} \mid g_{121}, g_{221}, g_{321} \in \mathfrak{p}_{v}\right\}, \\
& \bar{H}_{1}=\left\{g \in\left(G L(2)_{\mathcal{O}_{v} / \mathfrak{p}_{v}}\right)^{3} \mid g_{121}=g_{221}=g_{321}=0\right\}
\end{aligned}
$$

and if $v \in \mathfrak{M}_{\text {in }}$ then we define

$$
\begin{aligned}
& H_{2}=\left\{g \in K_{v} \mid g_{121} \in \tilde{\mathfrak{p}}_{v}, g_{221} \in \mathfrak{p}_{v}\right\} \\
& \bar{H}_{2}=\left\{g \in G L(2)_{\tilde{\mathcal{O}}_{v} / \tilde{\mathfrak{p}}_{v}} \times G L(2)_{\mathcal{O}_{v} / \mathfrak{p}_{v}} \mid g_{121}=0, g_{221}=0\right\}
\end{aligned}
$$

Note that

$$
\#\left(K_{v} / H_{1}\right)=\#\left(G_{\mathcal{O}_{v} / \mathfrak{p}_{v}} / \bar{H}_{1}\right)=\frac{\left(q_{v}^{2}-1\right)^{3}\left(q_{v}^{2}-q_{v}\right)^{3}}{\left(q_{v}-1\right)^{6} q_{v}^{3}}=\left(q_{v}+1\right)^{3}
$$

and

$$
\begin{aligned}
\#\left(K_{v} / H_{2}\right) & =\#\left(G_{\mathcal{O}_{v} / \mathfrak{p}_{v}} / \bar{H}_{2}\right)=\frac{\left(q_{v}^{4}-1\right)\left(q_{v}^{4}-q_{v}^{2}\right)\left(q_{v}^{2}-1\right)\left(q_{v}^{2}-q_{v}\right)}{\left(q_{v}^{2}-1\right)^{2} q_{v}^{2}\left(q_{v}-1\right)^{2} q_{v}} \\
& =\left(q_{v}^{2}+1\right)\left(q_{v}+1\right) .
\end{aligned}
$$

LeMmA 4.24. (1) If $v \in \mathfrak{M}_{\mathrm{sp}}$ and $g \in H_{1}$ then $g \mathscr{D}_{\ell}=\mathscr{D}_{\ell}$.

(2) If $v \notin \mathfrak{M}_{\mathrm{sp}}$ and $g \in H_{2}$ then $g \mathscr{D}_{\ell}=\mathscr{D}_{\ell}$.

Proof. A simple, direct calculation shows that elements of $H_{1}$ and $H_{2}$ preserve all the conditions for membership in $\mathscr{D}_{\ell}$, with the possible exception of the last. Consider (1) and suppose that $y=g x$ with $g \in H_{1}$ and $x \in \mathscr{D}_{\ell}$. Then $F_{y}(v)=$ $\operatorname{det}\left(g_{1}\right) \operatorname{det}\left(g_{2}\right) F_{x}\left(v g_{3}\right)$ and so $a_{1}(y)$ is equal to

$$
\operatorname{det}\left(g_{1}\right) \operatorname{det}\left(g_{2}\right)\left(a_{1}(x)\left[g_{311} g_{322}+g_{321} g_{312}\right]+2 a_{0}(x) g_{311} g_{321}+2 a_{2}(x) g_{312} g_{322}\right) .
$$

We must investigate the order of this number. Since $\operatorname{det}\left(g_{1}\right)$ and $\operatorname{det}\left(g_{2}\right)$ are units, they may be ignored. Both $g_{311}$ and $g_{322}$ are units and, since $g_{321} \in \mathfrak{p}_{v}, g_{311} g_{322}+g_{321} g_{312}$ is also a unit. Thus the order of $a_{1}(x)\left[g_{311} g_{322}+g_{321} g_{312}\right]$ equals $\ell$ if $\ell \leq m_{v}$ and is greater than or equal to $m_{v}+1$ if $\ell=m_{v}+1$. Also, $\operatorname{ord}_{k_{v}}\left(2 a_{0}(x) g_{311} g_{321}\right) \geq m_{v}+1$ and $\operatorname{ord}_{k_{v}}\left(2 a_{2}(x) g_{312} g_{322}\right) \geq m_{v}+1$, in the first case because $g_{321} \in \mathfrak{p}_{v}$ and in the second because $a_{2}(x) \in \mathfrak{p}_{v}$. It follows that the order of $a_{1}(y)$ is $\ell$ if $\ell \leq m_{v}$ and is greater than or equal to $m_{v}+1$ if $\ell=m_{v}+1$. Thus $y \in \mathscr{D}_{\ell}$, as required. Similar arguments apply in case (2).

Proposition 4.25. (1) Suppose $v \in \mathfrak{M}_{\mathrm{sp}}$ is dyadic. Then

$$
\begin{aligned}
\sum_{2 \leq \delta_{x, v}=2 \ell \leq 2 m_{v}} \operatorname{vol}\left(K_{v} x\right) & =\left(1-q_{v}^{-1}\right)^{2}\left(1-q_{v}^{-2}\right)^{3} q_{v}^{-\ell}, \\
\sum_{\delta_{x, v}=2 m_{v}+1} \operatorname{vol}\left(K_{v} x\right) & =\left(1-q_{v}^{-1}\right)\left(1-q_{v}^{-2}\right)^{3} q_{v}^{-\left(m_{v}+1\right)} .
\end{aligned}
$$

(2) Suppose $v \in \mathfrak{M}_{\text {in }}$ is dyadic. Then 


$$
\begin{aligned}
\sum_{\delta_{x, v}=2 \ell \leq 2 m_{v}} \operatorname{vol}\left(K_{v} x\right) & =\left(1-q_{v}^{-1}\right)^{2}\left(1-q_{v}^{-2}\right)\left(1-q_{v}^{-4}\right) q_{v}^{-\ell} \\
\sum_{\delta_{x, v}=2 m_{v}+1} \operatorname{vol}\left(K_{v} x\right) & =\left(1-q_{v}^{-1}\right)\left(1-q_{v}^{-2}\right)\left(1-q_{v}^{-4}\right) q_{v}^{-\left(m_{v}+1\right)} .
\end{aligned}
$$

Proof. Consider (1). We first show that $\mathscr{D}_{\ell} \subseteq \bigcup_{x} K_{v} x$, where $x$ runs through the standard orbital representatives for the orbits of type (sp $\mathrm{rm}$ ) whose corresponding fields have a fixed discriminant. As in the statement, the discriminant is related to $\ell$ by $\ell=\left\lfloor\left(\delta_{x, v}+1\right) / 2\right\rfloor$. By construction, if $y \in \mathscr{D}_{\ell}$ then $|P(y)|_{v}=q_{v}^{-\delta_{x, v}}$ and so if $x$ is the standard representative for the orbit corresponding to $k_{v}(y)$ then $|P(y)|_{v}=|P(x)|_{v}$. Since $y \in G_{k_{v}} x \cap V_{\mathcal{O}_{v}}$, the theory of omega sets implies that there exists $g \in \Omega_{x, v}$ such that $y=g x$. But then $|\chi(g)|_{v}=1$ and so $g \in \Omega_{x, v}^{1}=K_{v}$. That is, $y \in K_{v} x$.

Each one of the standard orbital representatives itself lies in $\mathscr{D}_{\ell}$ and so the $K_{v^{-}}$ translates of $\mathscr{D}_{\ell}$ cover $\bigcup_{x} K_{v} x$. In order to find the volume of $\bigcup_{x} K_{v} x$ it remains to determine the number of distinct $K_{v}$-translates of $\mathscr{D}_{\ell}$. Suppose that $g \in K_{v}$ and $g \mathscr{D}_{\ell} \cap \mathscr{D}_{\ell} \neq \varnothing$; say $x, y \in \mathscr{D}_{\ell}$ and $y=g x$. We shall show that $g \in H_{1}$. Since $F_{x}(v)$, $F_{y}(v)$ both reduce to unit scalar multiples of $v_{1}^{2}$ modulo $\mathfrak{p}_{v}$, the assumption implies that $g_{321} \in \mathfrak{p}_{v}$. Since $\left(1,1, g_{3}\right) \in H_{1}$, we may assume $g_{3}=1$. Let $x=\left(x_{i j k}\right)$ and $\bar{x}=\left(\bar{x}_{i j k}\right)$ be the reduction of $x$ modulo $\mathfrak{p}_{v}$. We define $\bar{g}_{i j k}, \bar{y}$ similarly. Then

$$
\left(g_{1}, g_{2}, 1\right) \bar{x}=\bar{y}=\left(*, \bar{x}_{211}\left(\begin{array}{ll}
\bar{g}_{111} \bar{g}_{211} & \bar{g}_{111} \bar{g}_{221} \\
\bar{g}_{121} \bar{g}_{212} & \bar{g}_{121} \bar{g}_{222}
\end{array}\right)\right) .
$$

Since $\bar{g}_{212} \neq 0$ or $\bar{g}_{222} \neq 0, \bar{g}_{121}=0$. This implies that $\bar{g}_{111} \neq 0$ and therefore $\bar{g}_{221}=0$. Thus $g \in H_{1}$, as claimed.

It follows from this and Lemma 4.24 that two $K_{v}$-translates of $\mathscr{D}_{\ell}$ are either disjoint or equal and that the number of them is $\#\left(K_{v} / H_{1}\right)=\left(q_{v}+1\right)^{3}$ by $(4.22)$. Using Proposition 4.19, the result follows. Similar arguments apply to prove the formula in case (2).

Proposition 4.26. If $v$ is not dyadic and $x$ has type ( $\mathrm{rm} \mathrm{rm} \mathrm{ur)} \mathrm{or} \mathrm{(rm} \mathrm{rm)*} \mathrm{then}$

$$
\operatorname{vol}\left(K_{v} x\right)=\frac{1}{2} q_{v}^{-1}\left(1-q_{v}^{-1}\right)\left(1-q_{v}^{-2}\right)^{2}
$$

Proof. Consider $\mathscr{D}$ in Definition 4.10(6). Obviously $\operatorname{vol}(\mathscr{D})=q_{v}^{-16}$. In both cases, $G_{x \mathcal{O}_{v} / \mathfrak{p}_{v}^{2}}^{\circ}$ consists of elements of the form $\left(A_{p}(c, d), *\right)$ where $c \in\left(\tilde{\mathcal{O}}_{v} / \tilde{\mathfrak{p}}_{v}^{4}\right)^{\times}, d \in \tilde{\mathcal{O}}_{v} / \tilde{\mathfrak{p}}_{v}^{4}$. So $\# G_{x \mathcal{O}_{v} / \mathfrak{p}_{v}^{2}}^{\circ}=\left(q_{v}-1\right) q_{v}^{7}$.

We show that $\left[G_{\bar{x} \mathcal{O}_{v} / \mathfrak{p}_{v}^{2}}: G_{x \mathcal{C}_{v} / \mathfrak{p}^{2}}^{\circ}\right]=2 q_{v}$. Suppose $g \in G_{\bar{x} \mathcal{O}_{v} / \mathfrak{p}_{v}^{2}}$. We consider the coordinates (2.19) again. Since $F_{x}(v)$ is a unit scalar multiple of $v_{1}^{2}$ modulo $\mathfrak{p}_{v}$, $g_{221} \equiv 0\left(\mathfrak{p}_{v}\right)$. Then since $g_{1}\left(\begin{array}{ll}1 & 0 \\ 0 & 0\end{array}\right)^{t} g_{1}^{\sigma}$ is a unit scalar multiple of $\left(\begin{array}{ll}1 & 0 \\ 0 & 0\end{array}\right), g_{121} \equiv 0\left(\tilde{\mathfrak{p}}_{v}\right)$. So $g_{111}, g_{122}, g_{211}, g_{222}$ are units. This implies that the right $G_{x \mathfrak{U}_{v} / \mathfrak{p}_{v}^{2}}^{\circ}$-coset of $g$ contains an element of the form $\left(\left(\begin{array}{ll}1 & 0 \\ u & t\end{array}\right), *\right)$. We use Lemma 4.4 again. By computation,

$$
\left(\left(\begin{array}{ll}
1 & 0 \\
u & t
\end{array}\right), 1\right) x=\left(\left(\begin{array}{cc}
0 & t^{\sigma} \\
t & \operatorname{Tr}_{\tilde{k}_{v} / k_{v}}\left(t u^{\sigma}\right)
\end{array}\right),\left(\begin{array}{cc}
1 & u^{\sigma} \\
u & \mathrm{~N}_{\tilde{k}_{v} / k_{v}}(u)-a_{2} \mathrm{~N}_{\tilde{k}_{v} / k_{v}}(t)
\end{array}\right)\right) .
$$


So $t=t^{\sigma}, \quad u=u^{\sigma}, \quad 2 t u=0, \quad$ and $a_{2}\left(t^{2}-1\right)-u^{2}=0$. Since $v \notin \mathfrak{M}_{\mathrm{dy}}, \quad u=0$, and $t \equiv \pm 1\left(\mathfrak{p}_{v}\right)$. Since $t \in\left(\mathcal{O}_{v} / \mathfrak{p}_{v}^{2}\right)^{\times}$, there are $2 q_{v}$ possibilities for $t$. Therefore, in both cases,

$$
\operatorname{vol}\left(K_{v} x\right)=q_{v}^{-16} \cdot \frac{\left(q_{v}^{2}-1\right)^{2}\left(q_{v}^{2}-q_{v}\right)^{2} q_{v}^{16}}{2\left(q_{v}-1\right) q_{v}^{8}}=\frac{1}{2} q_{v}^{-1}\left(1-q_{v}^{-1}\right)\left(1-q_{v}^{-2}\right)^{2}
$$

\section{Computation of $b_{x, v}$ at the infinite places.}

In this section we shall compute the constants $b_{x, v}$ when $v$ is an infinite place of $k$. We shall first review the definition of the constants $b_{x, v}$.

Let $v \in \mathfrak{M}_{\infty}$ and $x \in V_{k_{v}}^{\text {ss }}$ be a standard representative. Let $d g_{x, v}^{\prime \prime}$ be the measure on $G_{x k_{v}}^{\circ}$ defined in Section 2. We choose a left invariant measure $d g_{x, v}^{\prime}$ on $G_{k_{v}} / G_{x k_{v}}^{\circ}$ so that if $\Phi$ is a Schwartz-Bruhat function on $V_{k_{v}}$ then

$$
\int_{G_{k_{v}} / G_{x k_{v}}^{\circ}}\left|P\left(g_{x, v}^{\prime} x\right)\right|_{v}^{s} \Phi\left(g_{x, v}^{\prime} x\right) d g_{x, v}^{\prime}=\int_{G_{k_{v}}}|P(y)|_{v}^{s-2} \Phi(y) d y,
$$

where $d y$ is the Lebesgue measure if $v \in \mathfrak{M}_{\boldsymbol{R}}$ and $2^{8}$ times Lebesgue measure if $v \in \mathfrak{M}_{\boldsymbol{C}}$. Let $d g_{v}$ be the standard measure on $G_{k_{v}}$. Then there exists a constant $b_{x, v}>0$ such that $d g_{v}=b_{x, v} d g_{x, v}^{\prime} d g_{x, v}^{\prime \prime}$.

It is established in Section 7 [2] that the contribution of the orbit of $x$ to the local density is $b_{x, v}^{-1}|P(x)|_{v}^{2}$. Since $x$ is a standard representative, $|P(x)|_{v}=1$. It was proved in Proposition 5.23 [2] that if $y \in G_{k_{v}} x$ then $b_{x, v}=b_{y, v}$. So we are free to make use of any orbital representative to carry out this calculation and we shall take advantage of this freedom to simplify our task as much as possible. In the proof of each of the four propositions in this section we shall have to calculate the $8 \times 8$ Jacobian determinant associated with the map $g \rightarrow g x$ in some coordinate system. Each of these calculations was carried out using the MAPLE computer algebra package [4].

Before we begin, we recall a fact about the Haar measure on $G L(2)_{F}$ where $F=\boldsymbol{R}$ or $C$. As is usual, we shall take Lebesgue measure to be the standard measure on the real numbers and twice Lebesgue measure to be the standard measure on the complex numbers. If $g=\left(\begin{array}{ll}a_{11} & a_{12} \\ a_{21} & a_{22}\end{array}\right)$ then $d \mu(g)=d a_{11} d a_{12} d a_{21} d a_{22} /|\operatorname{det}(g)|_{F}^{2}$ defines a Haar measure on $G L(2)_{F}$ and it is well known that if $d g$ denotes our standard choice of Haar measure on this group then $d g=p_{F} d \mu(g)$ where $p_{F}=1 / \pi$ if $F=\boldsymbol{R}$ and $p_{F}=1 / 2 \pi$ if $F=\boldsymbol{C}$. For example if $F=\boldsymbol{R}$, using the usual paramatrization of each factor of the Iwasawa decomposition, all one has to do is to find the Jacobian of the map $\mathrm{O}(2) \times$ $T_{R} \times N_{R} \rightarrow G_{R}$ and the degree of this covering and this can be done easily. When $F=k_{v}$ we shall use the notation $p_{v}$ in place of $p_{F}$. We shall refer to $d \mu(g)$ as the coordinate measure on $G L(2)_{F}$.

Proposition 5.2. Let $v \in \mathfrak{M}_{\infty}$ and suppose that $x$ is a representative for the orbit of index (sp). Then

$$
b_{x, v}= \begin{cases}\frac{2}{\pi^{3}} & \text { if } v \in \mathfrak{M}_{\boldsymbol{R}} \\ \frac{1}{4 \pi^{3}} & \text { if } v \in \mathfrak{M}_{C}\end{cases}
$$


Proof. As noted above, it suffices to compute $b_{x, v}$ where $x=w$, the element introduced in (2.8). Let $d \mu_{v}$ be the product of the coordinate measures on each of the three factors of $G_{k_{v}}$ so that $d g_{v}=p_{v}^{3} d \mu_{v}(g)$.

Let $\mathscr{B}$ denote the set of $\left(g_{1}, g_{2}, g_{3}\right) \in G_{k_{v}}$ such that $g_{1}$ and $g_{2}$ lie in the big Bruhat cell. Then $\mathscr{B}$ is dense in $G_{k_{v}}$ and it is on this set that we shall carry out the comparison of measures. Any element $g$ of $\mathscr{B}$ may be written as

$$
g=\left({ }^{t} n\left(u_{1}\right) n\left(y_{1}\right) a\left(t_{11}, t_{12}\right),{ }^{t} n\left(u_{2}\right) n\left(y_{2}\right) a\left(t_{21}, t_{22}\right), g_{3} a\left(t_{11}^{-1} t_{21}^{-1}, t_{12}^{-1} t_{22}^{-1}\right)\right)
$$

with $g_{3}=\left(\begin{array}{ll}a_{11} & a_{12} \\ a_{21} & a_{22}\end{array}\right)$ and when $g$ is written in this form, $u_{i}, y_{i}$ and $a_{i j}$ for $i, j=1,2$ may be regarded as coordinates on $G_{k_{v}} / G_{x k_{v}}^{\circ}$. Note that the map

$$
k_{v}^{2} \times\left(k_{v}^{\times}\right)^{2} \ni\left(u, y, t_{1}, t_{2}\right) \rightarrow{ }^{t} n(u) n(y) a\left(t_{1}, t_{2}\right) \in G L(2)_{k_{v}}
$$

is injective. With respect to these coordinates, the Jacobian determinant of the map $g \rightarrow g x$ is found to be $\left|\operatorname{det}\left(g_{3}\right)\right|_{v}^{2}$. Note that this map is a double cover because $\left[G_{x k_{v}}: G_{x k_{v}}^{\circ}\right]=2$. Since $P(g x)=\chi(g) P(x)=\operatorname{det}\left(g_{3}\right)^{2} P(x)$ and $P(x)=1$, it follows that the pullback of the measure $d y /|P(y)|_{v}^{2}$ to $G_{k_{v}} / G_{x k_{v}}^{\circ}$ is $d g_{x, v}^{\prime}=(1 / 2) d u_{1} d y_{1} d u_{2} d y_{2}$. $d \mu_{3, v}\left(g_{3}\right)$, where $d \mu_{3, v}\left(g_{3}\right)$ denotes the coordinate measure on the third factor. (The measure has been divided by 2 because the map $g \rightarrow g x$ is a double cover and, in (5.1), the measure $d g_{x, v}^{\prime}$ was defined via an integral over $V_{k_{v}}$.) In (5.3), $t_{i j}$ may be regarded as coordinates on $G_{x k_{v}}^{\circ}$ and as such $d g_{x, v}^{\prime \prime}=\prod_{i, j=1,2} d t_{i j} /\left|t_{i j}\right|_{v}$. It follows from the definition and the remarks above that $d \mu_{v}(g)=p_{v}^{-3} b_{x, v} d g_{x, v}^{\prime} d g_{x, v}^{\prime \prime}$ and so all that remains is for us to determine the relationship between the coordinate measure and the measure $d u d y d t_{1}$. $d t_{2} /\left|t_{1} t_{2}\right|_{v}$ on the big cell inside $G L(2)$ when it is coordinatized as ${ }^{t} n(u) n(y) a\left(t_{1}, t_{2}\right)$. A simple Jacobian calculation shows that in fact these two measures are equal. This gives $(1 / 2) p_{v}^{-3} b_{x, v}=1$ or equivalently $b_{x, v}=2 p_{v}^{3}$. Using the remarks before the proof, this gives the stated values.

Proposition 5.4. Let $v \in \mathfrak{M}_{\boldsymbol{R}}$ and suppose that $x$ is a representative for the orbit of index (rm). Then

$$
b_{x, v}=\frac{1}{\pi^{2}}
$$

Proof. We shall again use $x=w$ as the orbital representative. Let $\tilde{v}$ be the complex place of $\tilde{k}$ which divides $v$. Let $d \mu_{v}$ be the product of the coordinate measures on two factors of $G_{k_{v}}$ so that $d g_{v}=p_{\tilde{v}} p_{v} d \mu_{v}(g)$.

We let $\mathscr{B}$ denote the set of $\left(g_{1}, g_{2}\right) \in G_{k_{v}}$ such that $g_{1}$ lies in the big Bruhat cell. An element of $\mathscr{B}$ may be written as

$$
g=\left({ }^{t} n(u) n(y) a\left(t_{1}, t_{2}\right), g_{2} a\left(\mathrm{~N}_{\tilde{k}_{\tilde{v}} / k_{v}}\left(t_{1}\right)^{-1}, \mathrm{~N}_{\tilde{k}_{\tilde{v}} / k_{v}}\left(t_{2}\right)^{-1}\right)\right)
$$

with $g_{2}=\left(\begin{array}{ll}a_{11} & a_{12} \\ a_{21} & a_{22}\end{array}\right)$ and when $g$ is written in this form, $u, y$ and $a_{i j}, i, j=1,2$ may be regarded as coordinates on $G_{k_{v}} / G_{x k_{v}}^{\circ}$. With respect to the real coordinates $\operatorname{Re}(u), \operatorname{Im}(u)$, $\operatorname{Re}(y), \operatorname{Im}(y), a_{i j}$, the Jacobian determinant of the map $g \rightarrow g x$ is found to be $4\left|\operatorname{det}\left(g_{2}\right)\right|_{v}^{2}$. Since the map is a double cover, $P(g \cdot x)=\operatorname{det}\left(g_{2}\right)^{2}$ and the canonical measures $d u$ and $d y$ are $d u=2 d \operatorname{Re}(u) d \operatorname{Im}(u)$ and $d y=2 d \operatorname{Re}(y) d \operatorname{Im}(y)$, it follows that 
the pullback of the measure $d y /|P(y)|_{v}^{2}$ to $G_{k_{v}} / G_{x k_{v}}^{\circ}$ is $d g_{x, v}^{\prime}=(1 / 2) d u d y d \mu_{2, v}\left(g_{2}\right)$, where $d \mu_{2, v}\left(g_{2}\right)$ denotes the coordinate measure on the second factor. In (5.5), $t_{j}$ may be regarded as coordinates on $G_{x k_{v}}^{\circ}$ and as such $d g_{x, v}^{\prime \prime}=\prod_{j=1,2} d t_{j} /\left|t_{j}\right|_{\tilde{v}}$. It follows from the definition and the remarks above that $d \mu_{v}(g)=p_{\tilde{v}}^{-1} p_{v}^{-1} b_{x, v} d g_{x, v}^{\prime} d g_{x, v}^{\prime \prime}$ and since the coordinate measure restricted to the big cell inside $G L(2)_{\tilde{k}_{\hat{v}}}$ is $d u d y d t_{1} d t_{2} /\left|t_{1} t_{2}\right|_{\tilde{v}}$, we have $b_{x, v}=2 p_{\tilde{v}} p_{v}$.

Proposition 5.6. Suppose that $v \in \mathfrak{M}_{\boldsymbol{R}}$ and that $x$ is a representative for the orbit of index (sp rm). Then

$$
b_{x, v}=\frac{2}{\pi^{3}}
$$

Proof. We shall use $x=w_{p}$, where $p(z)=z^{2}+1$, as the orbital representative for this orbit. The roots of $p$ are $\pm \sqrt{-1}$. Let $A_{p}(c, d)$ be as in (2.11). As we discussed in Lemma 2.12, any $g \in G_{x k_{v}}^{\circ}$ has the form $g=\left(A_{p}\left(c_{1}, d_{1}\right), A_{p}\left(c_{2}, d_{2}\right), A_{p}\left(c_{3}, d_{3}\right)\right)$ where

$$
c_{3}=\frac{c_{1} c_{2}-d_{1} d_{2}}{\left(c_{1}^{2}+d_{1}^{2}\right)\left(c_{2}^{2}+d_{2}^{2}\right)}, \quad d_{3}=\frac{-\left(c_{1} d_{2}+c_{2} d_{1}\right)}{\left(c_{1}^{2}+d_{1}^{2}\right)\left(c_{2}^{2}+d_{2}^{2}\right)} .
$$

The isomorphism $\theta$ from $G_{x k_{v}}^{\circ}$ to $H_{x k_{v}}=\left(\boldsymbol{C}^{\times}\right)^{2}$ may be taken as

$$
\theta\left(A_{p}\left(c_{1}, d_{1}\right), A_{p}\left(c_{2}, d_{2}\right), A_{p}\left(c_{3}, d_{3}\right)\right)=\left(c_{1}+\sqrt{-1} d_{1}, c_{2}+\sqrt{-1} d_{2}\right) .
$$

Recalling that the canonical measure on $\boldsymbol{C}^{\times}$is $d z /|z|_{C}$ where $d z$ is twice Lebesgue measure, we see that

$$
d g_{x, v}^{\prime \prime}=\frac{4 d c_{1} d d_{1} d c_{2} d d_{2}}{\left(c_{1}^{2}+d_{1}^{2}\right)\left(c_{2}^{2}+d_{2}^{2}\right)}
$$

For any $r$ and any $\kappa \in S O(2)$ we may find $c$ and $d$ such that $a(r, r) \kappa=A_{p}(c, d)$ and it follows from this and the Iwasawa decomposition that any $g \in G L(2)_{\boldsymbol{R}}$ may be expressed as $g=n(u) a(1, t) A_{p}(c, d)$. Thus any $g \in G_{k_{v}}$ may be expressed as

$$
g=\left(n\left(u_{1}\right) a\left(1, t_{1}\right) A_{p}\left(c_{1}, d_{1}\right), n\left(u_{2}\right) a\left(1, t_{2}\right) A_{p}\left(c_{2}, d_{2}\right), g_{3} A_{p}\left(c_{3}, d_{3}\right)\right)
$$

where $g_{3}=\left(\begin{array}{ll}a_{11} & a_{12} \\ a_{21} & a_{22}\end{array}\right)$. Then $a_{i j}, t_{j}$ and $u_{j}, i, j=1,2$ may be regarded as coordinates on $G_{k_{v}} / G_{x k_{v}}^{\circ}$. With respect to these coordinates, the Jacobian determinant of the map $g \rightarrow g x$ is found to be $4\left|t_{1}^{2} t_{2}^{2} \operatorname{det}\left(g_{3}\right)^{2}\right|_{v}$. Since the map is a double cover, $P(g x)=$ $\chi(g) P(x)=t_{1}^{2} t_{2}^{2} \operatorname{det}\left(g_{3}\right)^{2} P(x)$ and $P(x)=-4$ this shows that the pullback of the measure $d y /|P(y)|_{v}^{2}$ to $G / G_{x k_{v}}^{\circ}$ is

$$
d g_{x, v}^{\prime}=\frac{d u_{1} d t_{1} d u_{2} d t_{2}}{8\left|t_{1}^{2} t_{2}^{2}\right|_{v}} d \mu_{3, v}\left(g_{3}\right)
$$

where $d \mu_{3, v}\left(g_{3}\right)$ is the coordinate measure on the third factor. An easy Jacobian calculation shows that if $g=n(u) a(1, t) A_{p}(c, d)$ then the coordinate measure is $d u d t d c d d /\left|t^{2}\right|_{v}\left(c^{2}+d^{2}\right)$ and so, with $d \mu_{v}$ denoting the product of the coordinate measures on the three factors, $d \mu_{v}=2 d g_{x, v}^{\prime} d g_{x, v}^{\prime \prime}$. Since $d g_{v}=p_{v}^{3} d \mu_{v}(g)=2 p_{v}^{3} d g_{x, v}^{\prime}$. $d g_{x, v}^{\prime \prime}$, it follows that $b_{x, v}=2 p_{v}^{3}$. 
Proposition 5.7. Suppose that $v \in \mathfrak{M}_{\boldsymbol{R}}$ and that $x$ is a representative for the orbit of index $(\mathrm{rm} \mathrm{rm})^{*}$. Then

$$
b_{x, v}=\frac{1}{\pi^{2}}
$$

Proof. We again use $x=w_{p}$, where $p(z)=z^{2}+1$, as the orbital representative. We let $\tilde{v}$ be the complex place of $\tilde{k}$ dividing $v$. With $A_{p}(c, d)$ as in (2.11) and

$$
c_{2}=\frac{\left|c_{1}\right|_{\tilde{v}}-\left|d_{1}\right|_{\tilde{v}}}{\left|c_{1}^{2}+d_{1}^{2}\right|_{\tilde{v}}}, \quad d_{2}=\frac{-\left(c_{1} \bar{d}_{1}+\bar{c}_{1} d_{1}\right)}{\left|c_{1}^{2}+d_{1}^{2}\right|_{\tilde{v}}},
$$

any element of $G_{x k_{v}}^{\circ}$ has the form $g=\left(A_{p}\left(c_{1}, d_{1}\right), A_{p}\left(c_{1}, d_{2}\right)\right)$ where $c_{1}^{2}+d_{1}^{2} \neq 0$. The isomorphism $\theta$ from $G_{x k_{v}}^{\circ}$ to $H_{x k_{v}}=\left(\boldsymbol{C}^{\times}\right)^{2}$ may be taken as

$$
\theta\left(A_{p}\left(c_{1}, d_{1}\right), A_{p}\left(c_{2}, d_{2}\right)\right)=\left(c_{1}+d_{1} \sqrt{-1}, c_{1}-d_{1} \sqrt{-1}\right) .
$$

From this it follows that

$$
d g_{x, v}^{\prime \prime}=\frac{4 d c_{1} d d_{1}}{\left|c_{1}^{2}+d_{1}^{2}\right|_{\tilde{v}}}
$$

It was shown during the proof of Lemma 9.2 [2] that any matrix $\left(\begin{array}{ll}m_{11} & m_{12} \\ m_{21} & m_{22}\end{array}\right)$ in $G L(2)_{C}$ may be written in the form $n(u) a(1, t) A_{p}\left(c_{1}, d_{1}\right)$ provided that $m_{11}^{2}+m_{12}^{2} \neq 0$. Since the complement of the set of matrices satisfying this condition has measure zero it suffices to make the comparison of measures on the set of elements of $G_{k_{v}}$ whose first entry satisfies this condition. Any $g$ in this set may be expressed as

$$
g=\left(n(u) a(1, t) A_{p}\left(c_{1}, d_{1}\right), g_{2} A_{p}\left(c_{2}, d_{2}\right)\right)
$$

where $g_{2}=\left(\begin{array}{ll}a_{11} & a_{12} \\ a_{21} & a_{22}\end{array}\right)$. We may use $a_{i j}, i, j=1,2, \operatorname{Re}(u), \operatorname{Im}(u), \operatorname{Re}(t)$ and $\operatorname{Im}(t)$ as real coordinates on (a set of comeasure zero in) $G_{k_{v}} / G_{x k_{v}}^{\circ}$. With respect to these coordinates the Jacobian determinant of the map $g \rightarrow g x$ is $16|t|_{\tilde{v}}^{2}\left|\operatorname{det}\left(g_{2}\right)\right|_{v}^{2}$. This shows, as usual, that the pullback of the measure $d y /|P(y)|_{v}^{2}$ to $G_{k_{v}} / G_{x k_{v}}^{\circ}$ is

$$
d g_{x, v}^{\prime}=\frac{d u d t}{8|t|_{\tilde{v}}^{2}} d \mu_{2, v}\left(g_{2}\right)
$$

where $d \mu_{2, v}\left(g_{2}\right)$ is the coordinate measure on the second factor. (Note that $d u=2 d \operatorname{Re}(u) d \operatorname{Im}(u)$ and $d t=2 d \operatorname{Re}(t) d \operatorname{Im}(t)$ again.) An easy Jacobian calculation shows that if $g=n(u) a(1, t) A_{p}\left(c_{1}, d_{1}\right)$ then the coordinate measure is $d u d t d c_{1} d d_{1} /$ $|t|_{\tilde{v}}^{2}\left|c_{1}^{2}+d_{1}^{2}\right|_{\tilde{v}}$ and so, with $d \mu_{v}$ denoting the product of the coordinate measures on the two factors, $d \mu_{v}=2 d g_{x, v}^{\prime} d g_{x, v}^{\prime \prime}$. Since $d g_{v}=p_{v} p_{\tilde{v}} d \mu_{v}(g)=2 p_{v} p_{\tilde{v}} d g_{x, v}^{\prime} d g_{x, v}^{\prime \prime}$, it follows that $b_{x, v}=2 p_{v} p_{\tilde{v}}$.

Even though we made use of results obtained from the software package MAPLE in the above proofs, everything in this section could have been proved manually without undue difficulty. For example, in the proof of Proposition 5.2, the only place we used MAPLE was to determine the Jacobian of the map $g \rightarrow g x$. Using the invariance properties of the measures, one can easily prove that this is a constant multiple of 
$\left|\operatorname{det}\left(g_{3}\right)\right|_{v}^{2}$. To determine this constant we only have to compute the Jacobian of the above map at the identity element. At this point the Jacobian matrix is a fairly sparse $8 \times 8$ matrix and its determinant is easily found by hand. Indeed, the value of this constant was verified manually in this case. However, we chose not to include the details of these manual calculations in this paper.

\section{References}

[ 1] A. C. Kable and A. Yukie, Prehomogeneous vector spaces and field extensions, II, Invent. Math., 130 (1997), 315-344.

[2] A. C. Kable and A. Yukie, The mean value of the product of class numbers of paired quadratic fields, I, Tohoku Math. J., 54 (2002), 513-565.

[ 3 ] A. C. Kable and A. Yukie, The mean value of the product of class numbers of paired quadratic fields, III, to appear in J. Number Theory.

[4] Waterloo Maple Software, Maple V, Waterloo Maple Inc., Waterloo, Ontario, 1994.

[5] A. Weil, Basic number theory, Springer-Verlag, Berlin-Heidelberg-New York, 1974.

[6] D. J. Wright and A. Yukie, Prehomogeneous vector spaces and field extensions, Invent. Math., 110 (1992), 283-314.

\author{
Anthony C. KABLE \\ Department of Mathematics \\ Oklahoma State University \\ Stillwater OK 74078, U.S.A. \\ E-mail: akable@math.okstate.edu
}

\author{
Akihiko YUKIE \\ Mathematical Institute \\ Tohoku University \\ Sendai Miyagi, 980-8578 \\ Japan \\ E-mail: yukie@math.tohoku.ac.jp
}

